\title{
Revisão da tafoflora interglacial neocarbonífera de Monte Mor, SP (Subgrupo Itararé), nordeste da Bacia do Paraná
}

\author{
Sandra Eiko Mune ${ }^{1}$ \& Mary E. C. Bernardes-de-Oliveira ${ }^{2}$
}

\begin{abstract}
Resumo A tafoflora do sítio Volpe, em Monte Mor, registra uma vegetação desenvolvida numa das fases interglaciais, ocorridas durante a Glaciação Neopaleozóica, no Estado de São Paulo. Esta assembléia, ocorrente na porção mediano-basal do Subgrupo Itararé, é constituída predominantemente de Paranocladus, Ginkgophyllum, Brasilodendron, e subordinadamente de outros gêneros, tais como Bumbudendron, Leptophloeum, cf. Cyclodendron, Trizygia, Koretrophyllites, Paracalamites, Botrychiopsis, Nothorhacopteris, Noeggerathiopsis, Buriadia, Cordaicarpus, Samaropsis e Paranospermum. Através de estudos morfográfico e cuticular, foi possível relacionar a presença de Paranocladus dusenii Florin 1940 com sementes platispérmicas de Paranospermum cambuiense Ricardi-Branco 1997. Registraram-se também formas inéditas para a assembléia, tais como, Ginkgophyllum cf. G. diazii, G. cf. G. kidstonii, (?) Ginkgophyllum spathulifolia Anderson \& Anderson 1985, Cordaicarpus cesarii Gutierrez, Ganuza, Morel \& Arrondo 1992 e Samaropsis cf. S. cuerdai. Entre os megásporos, Sublagenicula brasiliensis (Dijkstra) Dybová-Jachowicz et al. 1979 é a espécie mais abundante. Registraram-se ainda, duas formas inéditas para a localidade: Banksisporites tenuis (Dijkstra 1955) Glasspool 2003 e B. vulgatus (Dijkstra 1955) emend. Glasspool 2003. A análise palinológica revelou um conteúdo diversificado, predominantemente esporofítico, com abundância dos gêneros Lundbladispora e Vallatisporites e de tipos subsidiários como Horriditriletes, e Calamospora, entre outros, além de grãos de pólen dos gêneros Plicatipollenites, Potonieisporites, Meristocorpus, Limitisporites, e Scheuringipollenites. Essa flora interglacial desenvolveu-se em condições paleoclimáticas frias e não rigorosas, em ambientes de planície de inundação e de planície deltaica.
\end{abstract}

Palavras-chave: Subgrupo Itararé, Neocarbonífero, Monte Mor (SP).

\begin{abstract}
Revision of the late carboniferous interglacial taphoflora of Monte Mor, SP (Itararé Subgroup), northeast of the Paraná Basin. The taphoflora of the Volpe ranch, in Monte Mor Municipality (SP), registers a vegetation that developed in an interglacial phase during the Late Paleozoic Glaciation in the São Paulo State. This taphoflora, occurring in the mid-basal portion of the Itararé Subgroup, is constituted mainly by Paranocladus, Ginkgophyllum, Brasilodendron, and subordinately by genera such as Bumbudendron, Leptophloeum, cf. Cyclodendron, Trizygia, Koretrophyllites, Paracalamites, Botrychiopsis, Nothorhacopteris, Noeggerathiopsis, Buriadia, Cordaicarpus, Samaropsis and Paranospermum. By morphographic and cuticular studies, it was possible to relate better the association of Paranocladus dusenii Florin 1940 with platyspermic seeds of Paranospermum cambuiense Ricardi-Branco 1997. It was registered not yet recognized forms for this taphoflora, as Ginkgophyllum cf. G. diazii, G. cf. G. kidstonii, (?) Ginkgophyllum spathulifolia Anderson \& Anderson 1985, Cordaicarpus cesarii Gutierrez, Ganuza, Morel \& Arrondo 1992 and Samaropsis cf. S. cuerdai. Among the megaspores, the species Sublagenicula brasiliensis (Dijkstra) Dybová-Jachowicz et al. 1979 is the most abundant. Two forms not yet registered for the locality were detected: Banksisporites tenuis (Dijkstra 1955) Glasspool 2003 and B.Vulgatus (Dijkstra 1955) emend. Glasspool 2003. The palynological analysis revealed a diversified content, mainly sporophytic, with significant presence of the genera Lundbladispora and Vallatisporites, and subsidiary types as Horriditriletes and Calamospora. Among the pollen grains, the genera Plicatipollenites, Potonieisporites, Meristocorpus, Limitisporites and Scheuringipollenites are present. This interglacial flora was developed in cool paleoclimatic conditions and in flood and deltaic plains areas.
\end{abstract}

Keywords: Itararé Subgroup, Late Carboniferous, Monte Mor (SP).

INTRODUÇÃO A ocorrência megaflorística do sítio Volpe (ex-sítio da Mina) no Município de Monte Mor (SP) registra-se na literatura geológica desde Barbosa \& Almeida (1949). Seu estudo sistemático foi iniciado por Millan (1972), em Tese de Doutoramento pelo Instituto de Geociências da USP. Nos anos subseqüentes, gradualmente, Millan publicou sobre os diferentes grupos vegetais componentes da tafoflora de Monte Mor: Millan (1974) - sobre coniferópsidas; Millan (1976 e 1977a) - sobre moldes medulares de esfenófitas; Millan (1978, 1979a) - sobre espécies de Rhacopteris e Botrychiopsis; Millan (1979b) - sobre sementes godvâni-

1 - Pós-Graduação, Instituto de Geociências, Universidade de São Paulo, S. Paulo, SP, Brasil. E-mail: smune@hotmail.com

2 - Centro de Pós-Graduação, Pesquisa e Extensão da Universidade Guarulhos, Guarulhos, SP; Instituto de Geociências, Universidade de São Paulo, São Paulo, SP, Brasil e pesquisadora do CNPq. E-mail: maryeliz@usp.br 
cas; Millan (1981) - sobre cf. Ginkgophyllum e Millan (1980, 1985) - sobre Lepidodendrales. Trabalhos de síntese sobre a tafoflora são publicados em Millan (1975), onde apresenta seus elementos componentes e seu significado no Gondwana Inferior do Brasil; Millan (1977b), que, ao dar um panorama geral do documentário fitofossilífero do Gondwana brasileiro, apresentou outra vez a listagem de componentes da tafoflora de Monte Mor e Millan (1987) que apresentou o estado d'arte do conhecimento desta tafoflora ao caracterizar o Piso Montemorense, fazendo extensa discussão sobre o significado biocronoestratigráfico de sua micro e macroflora. Alarcon \& Bernardes-de-Oliveira (1999), ao fazerem uma síntese do registro licofítico do Neopaleozóico da bacia do Paraná, propuseram sinonímia para os espécimes de Lepidodendrales de Monte Mor (SP).

Após quase 30 anos, dá-se seqüência aos estudos sobre a tafoflora do sítio Volpe, contribuindo para a complementação do seu conhecimento e dos dados paleobotânicos do Estado de São Paulo. O material estudado anteriormente (depositado no Museu Nacional do Rio de Janeiro) e o de novas coletas realizadas em 2001 e 2002, serviram de base para o trabalho de revisão desenvolvido como mestrado da autora sênior, sob orien- tação da Profa. Dra. Mary E.C. Bernardes-de-Oliveira, no IGc./USP, com apoio financeiro do Projeto Temático FAPESP 97/03639-8.

Este trabalho teve ainda como objetivo a utilização de uma sistemática mais moderna e, através de novas amostras, obter dados anatômicos extraídos de carbonificações visando identificação taxonômica mais precisa dos exemplares; propor um melhor posicionamento bioestratigráfico dessa tafoflora e possíveis interpretações tafonômicas, paleoecológicas, paleogeográficas e paleoclimáticas.

GEOLOGIA REgIONAL Na bacia do Paraná, a Superseqüência Gondwana I, de Milani et al. (1998), corresponde ao maior ciclo transgressivo-regressivo (Fig. 1). Litoestratigraficamente, corresponde aos grupos Tubarão e Passa Dois. O Grupo Tubarão, formado pelos subgrupos Itararé e Guatá, teve suas camadas geradas em condições glaciais, interglaciais e pós-glaciais, apresentando litoestratigrafia bastante complexa.

O Subgrupo Itararé apresenta basalmente a Formação Lagoa Azul, que é não aflorante, ocorrendo em regiões de SP, PR e MS. Essa formação é composta de arenitos sobrepostos por folhelhos e diamictitos. Na

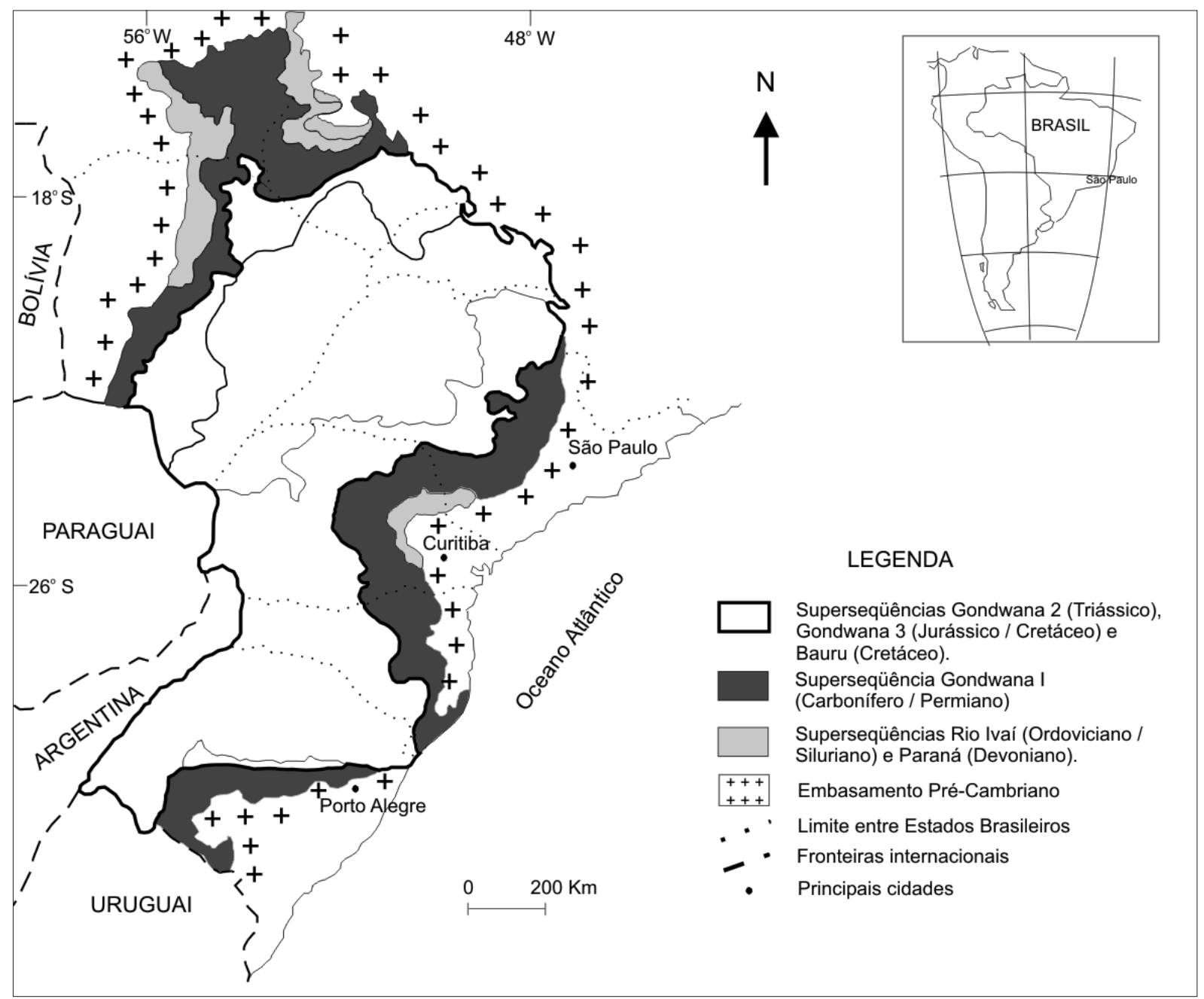

Figura 1- Superseqüências da bacia do Paraná (modificado de Milani et al. 1998). 
parte média do subgrupo, aparece a Formação Campo Mourão (França \& Potter, 1988), constituída de arenitos e diamictitos, ocorrendo no RS e SC. É constituída pelos membros Campo do Tenente e Rio Segredo. A Formação Taciba ocupa a porção superior do Subgrupo Itararé, ocorrendo em toda a bacia. É composta de folhelhos com intercalações arenosas e diamictitos. Subdivide-se nos membros Rio do Sul, que ocorre na parte sul da bacia do Paraná, e Membro Chapéu do Sol, aflorante nas porções central e norte da bacia, segundo Schneider et al. (1974) e Milani et al. (1994) (Fig. 2).

O Subgrupo Itararé atinge sua maior espessura no Estado de São Paulo, decrescendo gradativamente

\begin{tabular}{|c|c|c|c|c|c|c|c|}
\hline \multicolumn{4}{|c|}{ GEOCRONOLOGIA } & \multicolumn{4}{|c|}{ UNIDADES } \\
\hline \multicolumn{2}{|c|}{ Período } & Época & Idade & $\begin{array}{l}\text { Sub- } \\
\text { Grupo }\end{array}$ & & Formação & Membro \\
\hline \multirow{6}{*}{\multicolumn{2}{|c|}{ 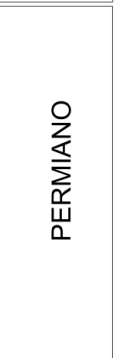 }} & \multirow{6}{*}{ 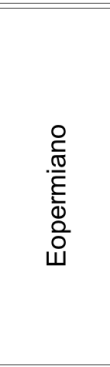 } & Kunguriano & \multirow{2}{*}{$\underset{0}{\stackrel{5}{5}}$} & \multirow{2}{*}{ 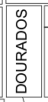 } & \multirow{2}{*}{$\begin{array}{c}\text { Palermo } \\
\text { Rio Bonito }\end{array}$} & Sidrónoli \\
\hline & & & Artinskiano & & & & Triunfo \\
\hline & & & \multirow{4}{*}{$\begin{array}{c}\text { Sakmariano } \\
\text { Asseliano }\end{array}$} & \multirow{7}{*}{ 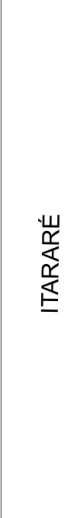 } & \multirow{7}{*}{$\begin{array}{l}\frac{\pi}{2} \\
\frac{1}{0} \\
\frac{\pi}{\pi} \\
\frac{\pi}{0} \\
\frac{0}{3} \\
\frac{1}{2}\end{array}$} & \multirow{4}{*}{ 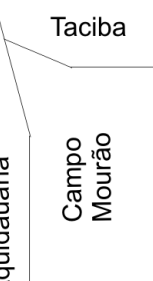 } & 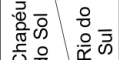 \\
\hline & & & & & & & Rio Segredo \\
\hline & & & & & & & \\
\hline & & & & & & & $\begin{array}{l}\text { Campo do } \\
\text { Tenente }\end{array}$ \\
\hline \multirow{3}{*}{ 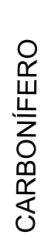 } & \multirow{3}{*}{ 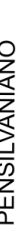 } & $\begin{array}{l}\text { Estefa- } \\
\text { niano }\end{array}$ & & & & & $\bar{\Omega}$ \\
\hline & & $\stackrel{\circ}{\frac{1}{\infty}}$ & & & & 胥 & $\frac{\sqrt[0]{\pi}}{\stackrel{\pi}{\circ}}$ \\
\hline & & $\begin{array}{l}\text { 垔 } \\
\stackrel{5}{3} \\
\stackrel{0}{3}\end{array}$ & & & & 茍 & 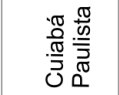 \\
\hline
\end{tabular}

Figura 2 - Esquema litoestratigráfico do Grupo Tubarão (modificado de Milani et al. 1994). para o sul. A litologia mais comum são os arenitos. Estes ocorrem isoladamente ou associados a diamictitos, aparecendo, com menor freqüência ritmitos e conglomerados. Em algumas localidades de São Paulo (Buri, Itapeva, Monte Mor e Cerquilho entre outras) ocorrem horizontes com camadas finas de carvão intercaladas nesses sedimentos, interpretadas como correspondendo a fases interglaciais por Bernardes-de-Oliveira et al (2005), (Fig. 3).

\section{LOCALIZAÇÃO GEOGRÁFICA E DADOS GE-} OLÓGICOS DA ÁREA O sítio Volpe dista cerca de $3 \mathrm{Km}$ da rodovia SP-101 (Campinas-Capivari), seguindo-se por uma estrada secundária, na área N-NE do Município de Monte Mor, limite com o Município de Sumaré. A localidade corresponde a uma mina abandonada de carvão (Fig. 4).

$\mathrm{O}$ afloramento situa-se na margem esquerda de um córrego seco (latitude 22 $54^{\prime} 55^{\prime}$ 'S e longitude $47^{\circ} 17^{\prime} \mathrm{W}$ ). Apresenta basalmente uma fina camada de carvão de aproximadamente $20 \mathrm{~cm}$ de espessura (Fig. $5)$.

Conforme observações de campo, os níveis fitofossilíferos de coleta posicionam-se, estratigraficamente, na capa e lapa do carvão, na porção basal de uma seqüência de lamitos acinzentados, carvões, arenitos finos e arcosianos. Uma camada espessa de diamictito se sobrepõe às anteriores, indicando a instalação de um novo ciclo glacial (Fig. 5).

Esse pacote sedimentar constitui parte da anteriormente designada, Formação Itu por Barbosa \& Ameida (1949:8-9) e seu tilito/diamictito superior corresponderia ao, então denominado, Membro Elias Fausto. Posteriormente, os diamictitos superiores, com grandes blocos erráticos ocorrentes na área do Município de Monte Mor, próximo ao sítio Volpe, foram pro-

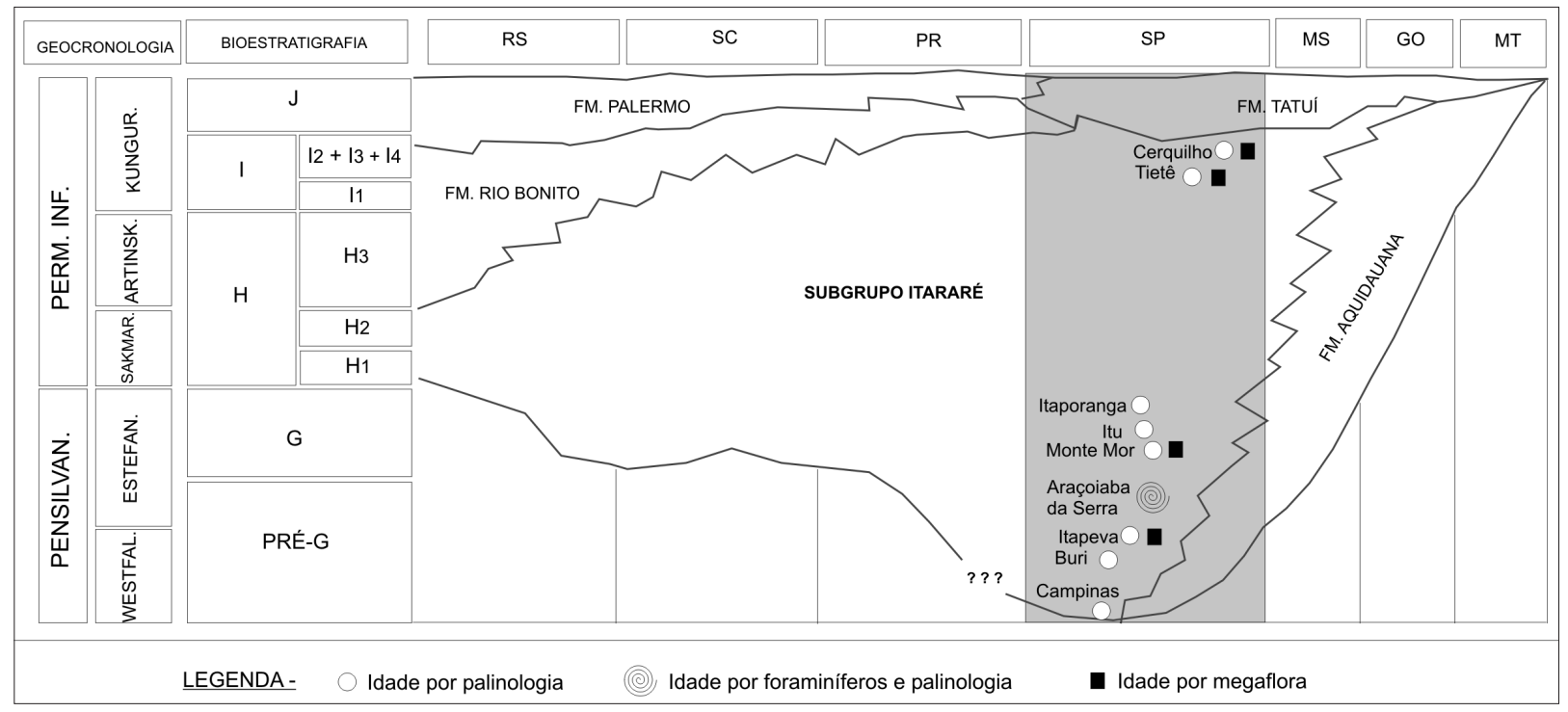

Figura 3 - Cronoestratigrafia do Grupo Tubarão (SE-NW) exibindo as grandes espessuras do Subgrupo Itararé no Estado de São Paulo e posicionando suas floras, com base em zoneamentos propostos por vários autores. Modificado de Bernardes-de-Oliveira et al. (2005), com base em Petri \& Souza (1993). 


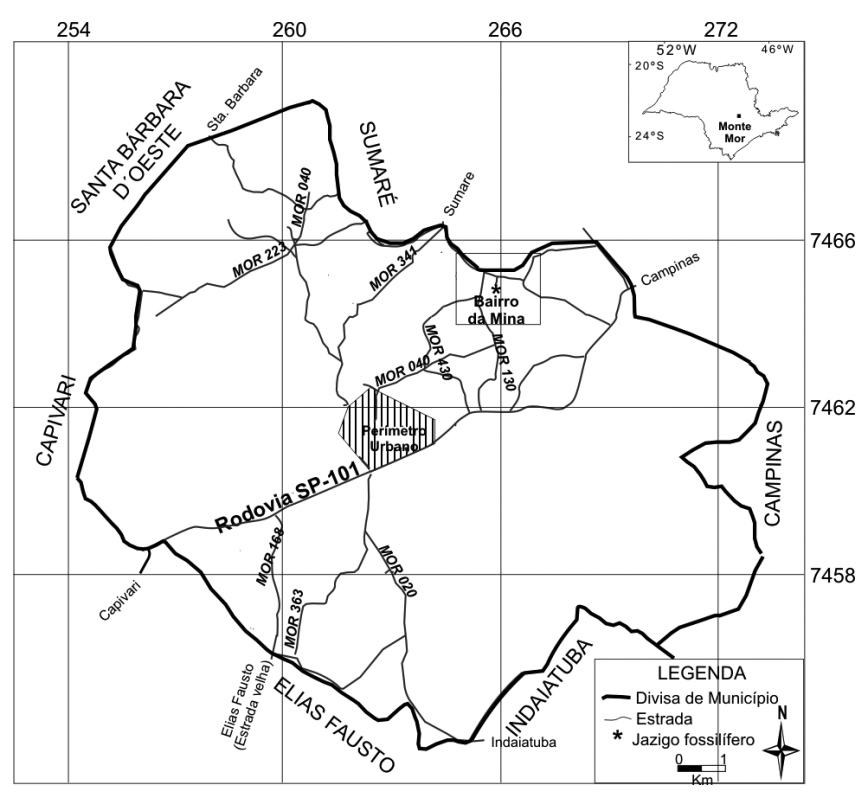

Figura 4 - Localização geográfica do Município de Monte Mor (SP) e da área de procedência dos fósseis (Fonte: Prefeitura Municipal de Monte Mor, SP).

movidos à categoria de Formação Elias Fausto por Barbosa \& Gomes (1958), incluída no Subgrupo Itararé.

Souza Filho (1986) elaborando um mapeamento geológico (litoestratigráfico, estrutural e faciológico) do Subgrupo Itararé, na quadrícula de Campinas (SP), que abrange o Município de Monte Mor, reconheceu sete unidades litoestratigráficas informais: Unidade I arenitos em granodecrescência ascendente; Unidade II - lamitos; Unidade III - lamitos e diamictitos; Unidade IV - arenitos; Unidade V - diamictitos e arenitos; Unidade VI - arenitos com marcas onduladas e Unidade VII - diamictitos vermelhos.

Segundo esse autor (1986:32-35), os níveis carbonosos, na área de Monte Mor, estariam incluídos na Unidade IV (constituída de vários corpos de arenitos que ocorrem em diferentes posições estratigráficas dentro da Unidade III) (Figs. 6 e 7). Os principais termos litológicos da Unidade IV seriam os arenitos conglomeráticos e os arenitos médios e finos, acompanhados de corpos conglomeráticos, lamitos, e carvão. As litofácies aí reconhecidas foram arenitos com estratificação cruzada tabular e acanalada, arenitos muito finos, arenitos com marcas onduladas, conglomerados maciços e com estratificação cruzada, lamitos com carvão e lamitos arroxeados.

Ainda conforme Souza Filho (1986), a área de Monte Mor estaria incluída, geneticamente, dentro do Sistema Supraglacial de Base úmida Elias Fausto, que compreenderia uma fácies de lobos deltaicos com arenitos com marcas onduladas e estratificação cruzada, envolvendo depósitos de frente deltaica proximal ou planície de maré e canais fluviais de planície deltaica, na base, sotoposta a uma fácies supraglacial de til de fluxo de ablação e uma fácies subglacial com tilitos de alojamento e pavimentos de clastos. Dentro da Fácies de Lobo Deltaico de Monte Mor, os lamitos com car-

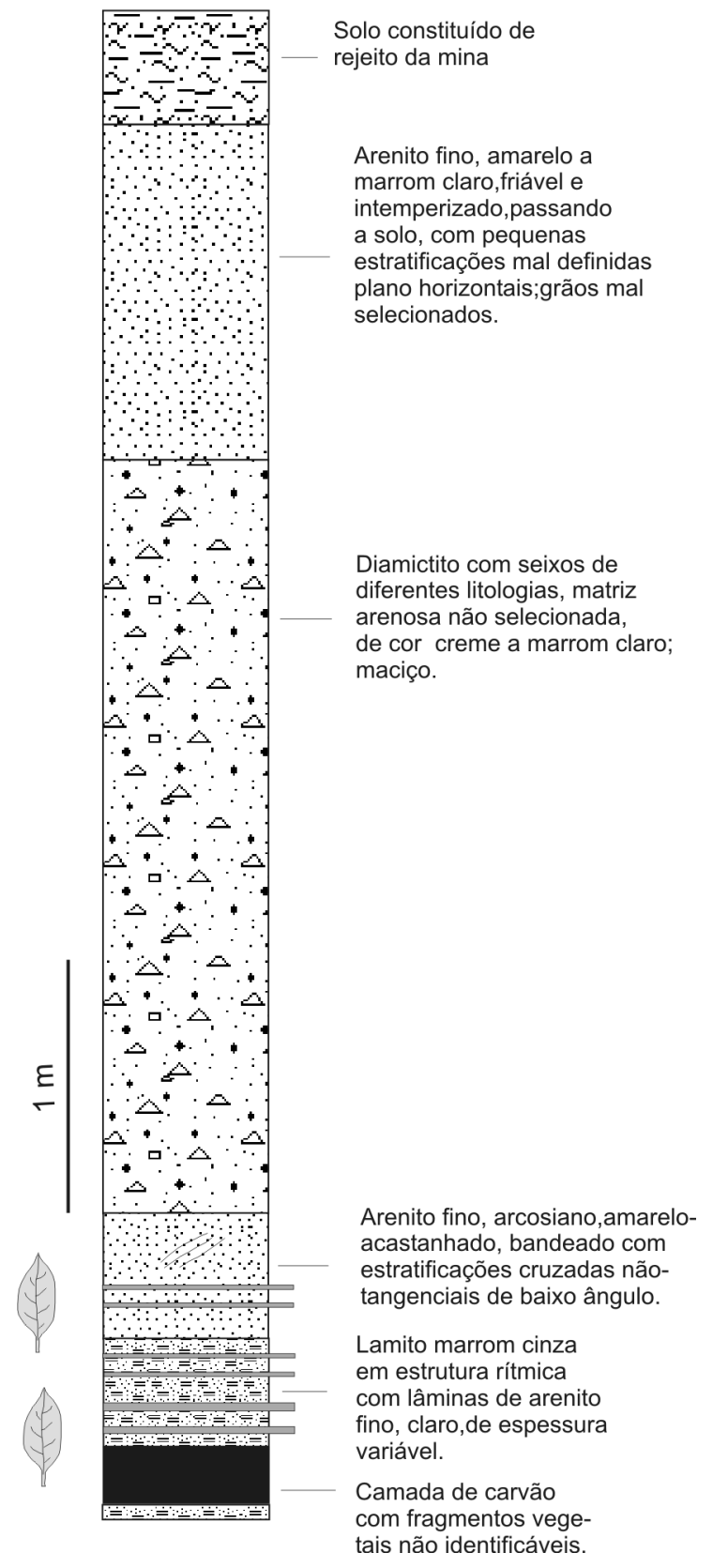

Figura 5 - Perfil geológico esquemático do afloramento do sitio Volpe.

vão, arenitos lamíticos e lamitos acinzentados, ricos em matéria orgânica e carvão, com estrutura maciça de flaser e lenticulares, siltitos com restos vegetais e fósseis de água doce representariam depósitos por decantação em condições restritas, ricas em matéria orgânica. Essa deposição ocorreria dentro de ambiente continental de planície de inundação, quase no final de uma seqüência de ambientes de prodelta, frente deltaica, praia, planície de inundação e dunas antes da instalação de novo ciclo glacial.

MATERIAL E MÉTODOS O material estudado é composto pela coleção de fósseis de Monte Mor coletada, estudada e depositada no Museu Nacional do Rio de Janeiro (UFRJ), por Millan (1972) e pelo material resultante de novas coletas, depositado na Coleção 


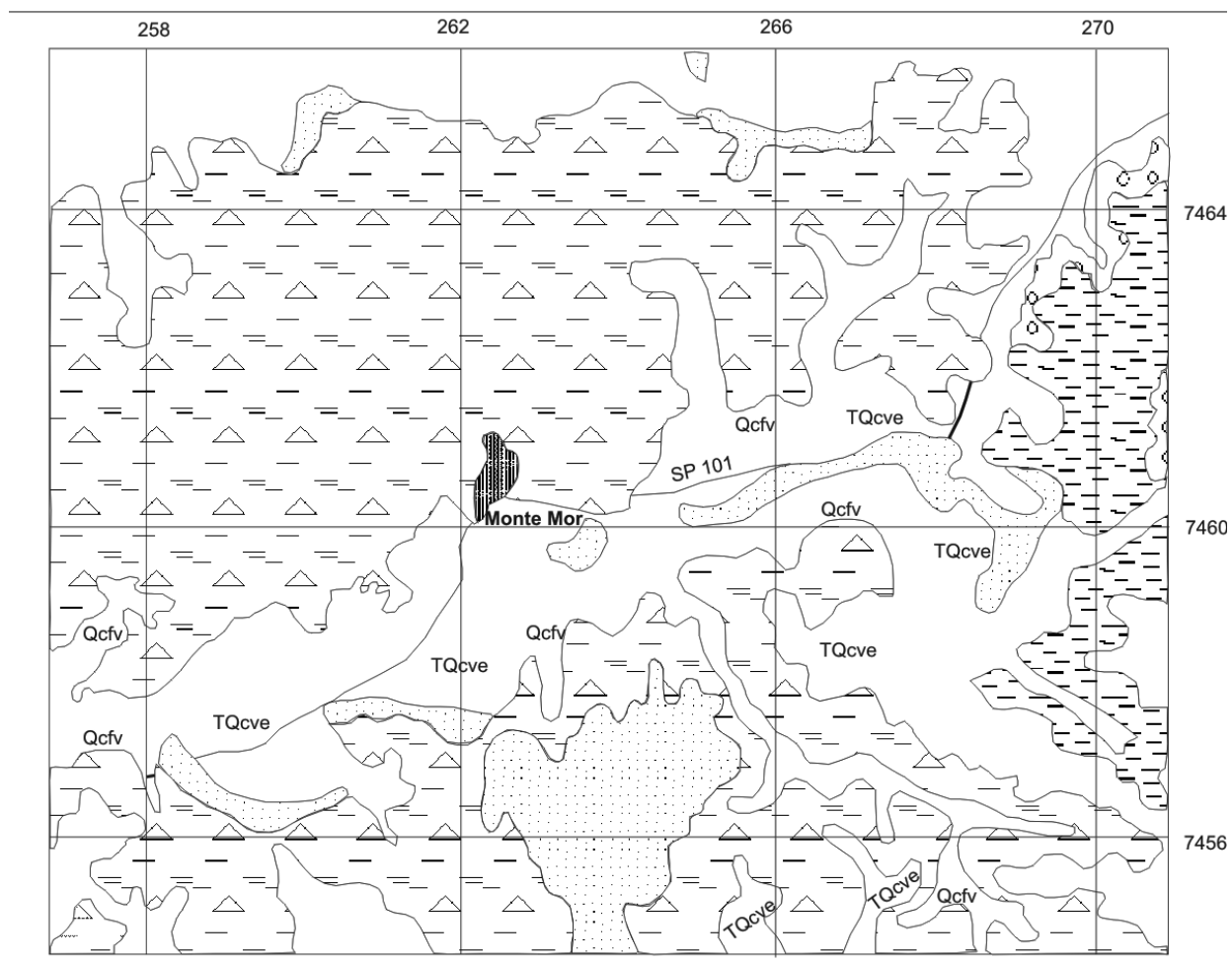

Legenda

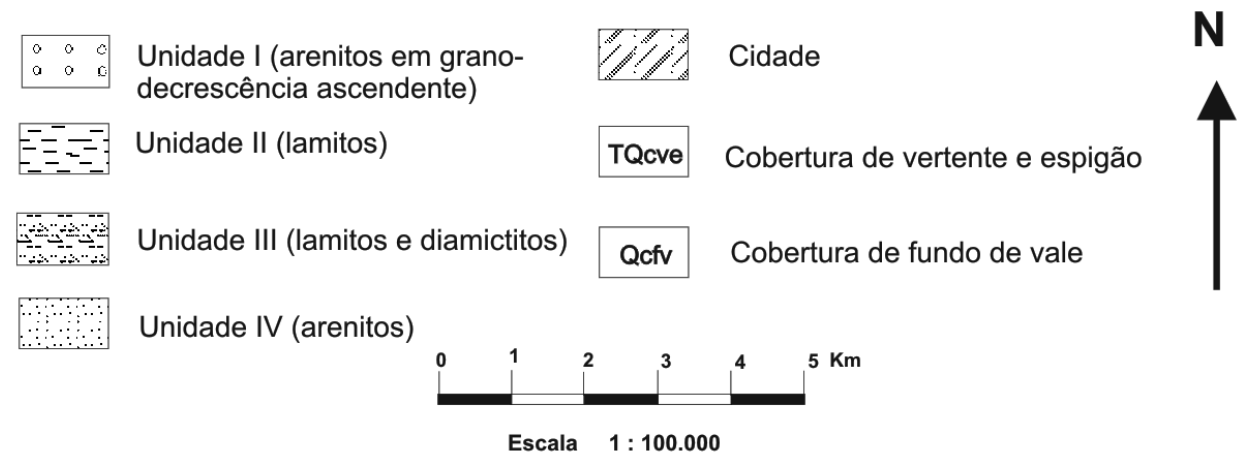

Figura 6 - Mapa geológico da área de Monte Mor, extraído de Campinas, de Souza Filho (1986).

Científica do Instituto de Geociências (USP). (Tabs. 1, 2 e 3$)$

Em campo, foi obedecido o procedimento normal de coleta e acondicionamento. Alguns blocos maiores foram coletados para serem abertos em laboratório. Medidas de direção preferencial do agente transportador dos fósseis foram tomadas localmente.

Em laboratório foi feita a preparação mecânica, consistindo na limpeza e numeração dos macrofósseis. A seguir, foram examinados e mensurados sob estereomicroscópio Carl Zeiss Stemi SV6 do Laboratório de Paleobotânica e Palinologia do GSA, IGc/USP. Também foram desenhados, sob câmara clara e fotomacro e fotomicrografados para documentação gráfica, descritos e comparados com a bibliografia pertinente. Posteriormente, foram realizados procedimentos específicos para obtenção de cutícula de macro e mesofósséis (sementes), utilizando-se o método de preparação química de Fittipaldi \& Rösler (1978) para plantas paleozói- cas. Optou-se pela maceração em bloco em solução de Schulze (ác. nítrico mais solução saturada de clorato de potássio), visto a película carbonosa estar quase sempre fortemente aderida à matriz rochosa. Os megásporos, extraídos da rocha utilizando-se HF, foram separados, identificados e fotografados em estereomicroscópio e sob M.E.V. Para os microfósseis (palinomorfos) foi utilizado o tratamento químico recomendado para amostras do Paleozóico, descrito por Quadros \& Mello (1987) e Souza (2000). Os palinomorfos foram analisados em microscópio óptico Carl Zeiss Axiophot II, fotografados por câmera digital e mensurados utilizandose o software Axiovision da Zeiss.

PRINCIPAIS RESULTADOS DA REVISÃO Após a finalização deste estudo, obteve-se melhor caracterização dos componentes fitofossilíferos que compõem a tafoflora interglacial neocarbonífera de Monte Mor (sítio Volpe), com base em identificações taxonômicas 
mais recentes realizadas na bacia do Paraná e na Argentina e em material cuticular. Também o ambiente deposicional no qual fossilizou-se e o contexto paleoecológico da paleoflora foram melhor caracterizados. Sendo assim, alguns resultados e conclusões podem aqui serem consignados.

Quanto à composição A assembléia fitofossilífera de Monte Mor apresenta uma predominância de plantas esporofíticas representadas por esfenófitas e licófitas e um teor menor de macrofósseis gimnospérmicos dentre os quais as coníferas (Voltziales) têm uma participação marcante (Fig. 8).

A análise deste material macrofossilífero resultou, parcialmente, em novas combinações genéricas, sinonímias e outras designações específicas (Tab. 4) que serão objeto de futuras publicações. Alguns desses elementos macroflorísticos podem ser visualizados nas

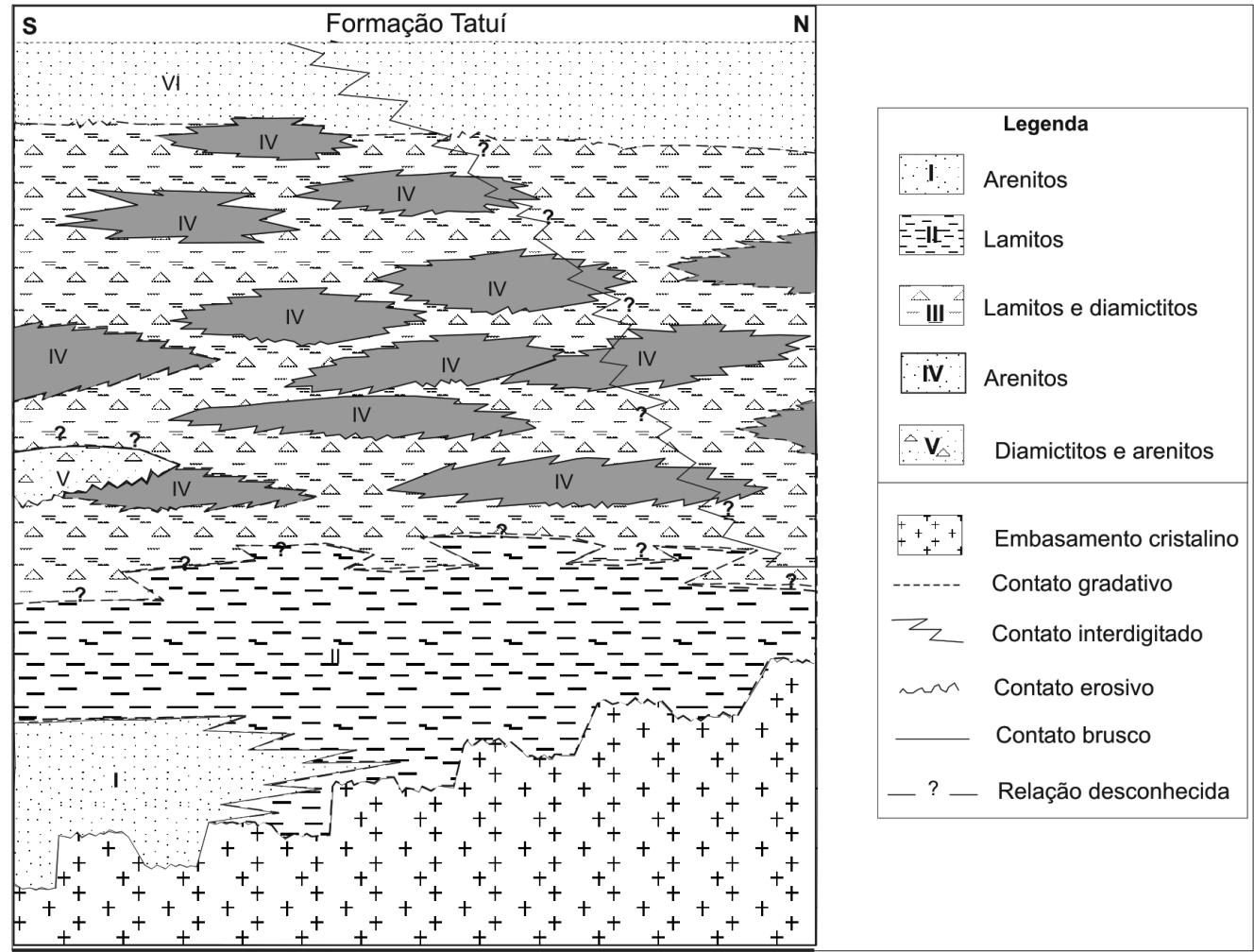

Figura 7 - Carta litoestratigráfica da região de Campinas evidenciando os corpos arenosos incluidos na Unidade III (segundo Souza Filho, 1986).

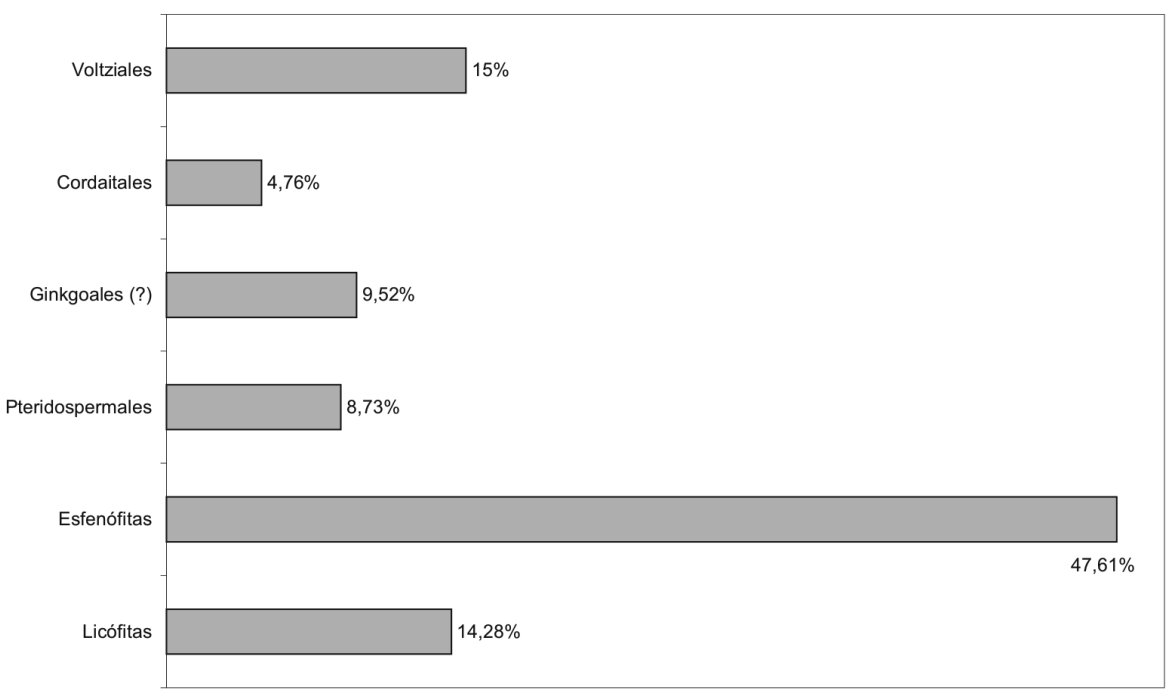

Figura 8 - Gráfico qualitativo/quantitativo dos grupos taxonômicos de macrofitofósseis da assembléia. 
Tabela 1 - Lista de taxa dos macrofitofósseis componentes da Tafoflora de Monte Mor (SP).

\begin{tabular}{|c|c|}
\hline \multicolumn{2}{|c|}{ Macrofitofósseis } \\
\hline Reino PLANTAE & $\begin{array}{l}\text { Paracalamites montemorensis Millan } 1977 \text { emend. } \\
\text { Zampirolli \& Bernardes-de-Oliveira } 2000\end{array}$ \\
\hline Divisão TRACHAEOPHYTA & Paracalamites sp \\
\hline Classe LYCOPSIDA & Classe GYMNOSPERMOPSIDA \\
\hline Ordem LEPIDODENDRALES & Ordem PTERIDOSPERMALES (?) \\
\hline Gênero Bumbudendron Archangelsky, Azcuy \& Wagner 1981 & $\begin{array}{l}\text { Gênero Botrychiopsis Kurtz 1895a emend. Archangelsky \& } \\
\text { Arrondo } 1971\end{array}$ \\
\hline $\begin{array}{l}\text { Bumbudendron millanii (Arrondo \& Petriella) Arrondo \& } \\
\text { Petriella } 1985\end{array}$ & Botrychiopsis cf. B. plantiana Archangelsky \& Arrondo 1971 \\
\hline $\begin{array}{l}\text { Bumbudendron cf. B. Paganzianum Archangelsky, Azcuy \& } \\
\text { Wagner } 1981\end{array}$ & Cf. Botrychiopsis sp \\
\hline Gênero Leptophloeum Dawson 1861 & Gênero Nothorhacopteris Archangelsky 1983 \\
\hline $\begin{array}{l}\text { Leptophloeum cf. L. sanctae-helenae Anderson \& Anderson } \\
1985\end{array}$ & Cf. Nothorhacopteris sp A \\
\hline Gênero Brasilodendron Chaloner, Leistkow \& Hill 1979 & Cf. Nothorhacopteris sp B \\
\hline $\begin{array}{l}\text { Brasilodendron pedroanum (Carruthers) Chaloner, Leistkow } \\
\text { \& Hill } 1979\end{array}$ & Ordem GINKGOALES (?) \\
\hline Aff. Brasilodendron $\mathrm{sp}$ & $\begin{array}{l}\text { MORFOGÊNERO DE POSIÇÃO INCERTA (sensu Seward, } \\
\text { 1918) }\end{array}$ \\
\hline Gênero Cyclodendron Kräusel 1934 & Gênero Ginkgophyllum Saporta 1875 \\
\hline Cf. Cyclodendron $\mathrm{sp}$ & $\begin{array}{l}\text { Ginkgophyllum cf. G. diazii Archangelsky \& Arrondo emend. } \\
\text { Archangelsky \& Leguizamon } 1980\end{array}$ \\
\hline Classe SPHENOPSIDA & $\begin{array}{l}\text { Ginkgophyllum cf. G. kidstonii (Seward) emend. Anderson \& } \\
\text { Anderson } 1985\end{array}$ \\
\hline Ordem SPHENOPHYLLALES & (?) Ginkgophyllum spathulifolia Anderson \&Anderson 1985 \\
\hline Gênero Trizygia Royale 1839 & Ordem CORDAITALES \\
\hline Trizygia $\mathrm{sp}$ & $\begin{array}{l}\text { Gênero Noeggerathiopsis Feistmantel emend. McLoughlin \& } \\
\text { Drinnan } 1996\end{array}$ \\
\hline Sphenophyllales indeterminadas & $\begin{array}{l}\text { Noeggerathiopsis cf. N. hislopii (Bunbury) Feistmantel emend. } \\
\text { McLoughlin \& Drinnan } 1996\end{array}$ \\
\hline Ordem EQUISETALES & Ordem VOLTZIALES \\
\hline Gênero Koretrophyllites Radczenko 1955 & Gênero Paranocladus Florin 1940 \\
\hline Koretrophyllites sp & Paranocladus dusenii Florin 1940 \\
\hline Gênero Paracalamites Zalessky 1927 & Gênero Buriadia Seward \& Sahni 1920 \\
\hline $\begin{array}{l}\text { Paracalamites australis Rigby } 1966 \text { emend. Rigby } 1969 \\
\text { emend. Zampirolli \& Bernardes-de-Oliveira } 2000 \\
\text { Paracalamites levis Rigby } 1966 \text { emend. Zampirolli \& } \\
\text { Bernardes-de-Oliveira2000 }\end{array}$ & $\begin{array}{l}\text { Buriadia aff. B. heterophylla (Feistmantel) Seward \& Sahni } \\
1920 \text { emend. Singh, Rothwell, Mapes \& Chandra } 2003\end{array}$ \\
\hline
\end{tabular}

figuras 9 (B-N) e $10(\mathrm{~A}-\mathrm{H})$.

O conteúdo mesoflorístico referente a sementes incertae sedis apresenta uma predominância extrema de Paranospermum (gênero de semente com afinidade atribuída a Paranocladus), cuja representatividade na assembléia é, de certa forma, corroborada pelo conteúdo macrofossilífero, figura 10 (K-L) e tabela 2. Quan- to ao conteúdo referente a megásporos, ressalta-se que os mais freqüentes se identificam como Sublagenicula brasiliensis (Dijkstra) Dybová-Jachowicz et al. 1979, figura $10(\mathrm{M})$ e tabela 2, espécie que apresenta, por associação, afinidade com o gênero Brasilodendron, coincidentemente, uma das formas de licófitas mais representativas na assembléia. Formas como Lagenois- 
Tabela 2 - Lista de taxa dos mesofitofósseis componentes da Tafoflora de Monte Mor (SP).

Classificação de sementes platispérmicas (Incertae sedis), segundo Mune (2005)

I - Com testa estreita ou sem testa

Gênero Cordaicarpus Geinitz 1862

I a - com crista mediana

Grupo 1 - sem outros caracteres morfológicos

1.1 - Cordaicarpus barbosanus Millan 1977

I b - sem uma crista mediana

Grupo 3 - ápice com sinus

3. 1 - Cordaicarpus cesarii Gutierrez, Ganuza, Morel \& Arrondo 1992

3. 4 - Cordaicarpus sp

II - com testa ampla e diferenciada

II a - com crista mediana

Grupo 1 - com sinus num dos lados

1.8 - Paranospermum cambuiense Ricardi-Branco 1997

1.9 - Paranospermum millanianum nov. sp.

1.10 - Samaropsis sp

II. b - sem crista mediana

II b. 1 - de contorno verticalmente oval / elíptico

Grupo 2 - sarcotesta com sinus num dos lados

1.4 - Samaropsis barcellosa (White) Seward 1917

Grupo 3 - sarcotesta com sinus em ambos os lados

1.1 - Samaropsis cf. S. cuerdai Gutierrez, Ganusa, Morel \& Arrondo 1992

II b. 3 - forma horizontalmente oval / elíptica

Grupo 2 - sarcotesta com sinus

2. 5 - Samaropsis aff. S. seixasii (White) Seward 1917

Classificação de Megásporos de Monte Mor, segundo Mune (2005)

Anteturma SPORITES H. Potonié 1893

Turma TRILETES (Reinsch) Potonié \& Kremp 1954

Subturma AZONOTRILETES Lüber 1935

Infraturma LAEVIGATI (Bennie \& Kidston, 1886) Potonié 1956

Gênero Calamospora Schopf, Wilson \& Bentall 1944

Suprasubturma LAGENOTRILETES Potonié \& Kremp 1957

Subturma GULATI Bharadwaj 1957

Gênero Banksisporites Dettmann 1961

Banksisporites endosporitiferus (Singh) Tewari \& Maheshwari 1992 emend. Glasspool 2003

Banksisporites tenuis (Dijkstra 1955 b) Glasspool 2003

Banksisporites vulgatus (Dijkstra 1955 b) emend.Glasspool 2003

Banksisporites indicus (Singh 1953) emend.Glasspool 2003

Banksisporites labiosus (Dijkstra 1955 b) Glasspool 2003

Gênero Lagenoisporites Potonié et Kremp emend. Dybová-Jachowicz et al. 1979

Lagenoisporites scutiformis Trindade 1970

Gênero Sublagenicula Dybová-Jachowicz, Jachowicz, Karczewska, Lachkar, Loboziak, Piérart, Turnau \& Zoldani 1979

Sublagenicula brasiliensis (Dijkstra) Dybová-Jachowicz, Jachowicz, Karczewska, Lachkar, Loboziak, Piérart,

Turnau \& Zoldani 1979

Sublagenicula tripartites (Trindade 1970) nov. Com.

Gênero Setosisporites (Ibrahim 1933) Potonié \& Kremp 1954 emend. Dybová-Jachowicz, Karczewska, Lachkar, Loboziak, Piérart,Turnau \& Zoldani 1979

Setosisporites $\mathrm{sp}$ 
Tabela 3 - Lista de taxa dos microfitofósseis componentes da Tafoflora de Monte Mor (SP).

\section{Palinomorfos}

Anteturma ProximegerminAnTes Potonié 1970

Turma TrILETES Reinsch emend. Dettmann 1963

Suprasubturma ACAVATITRILETES Lüber emend. Dettmann 1963

Subturma Azonotriletes Lüber emend. Dettmann 1963

Infraturma Laevigati Bennie \& Kidston emend. Potonié 1970 Gênero Leiotriletes Naumova emend. Potonié \& Kremp 1954 Leiotriletes virkkii Tiwari 1965

Leiotriletes gracilis Menéndez 1965

Gênero Calamospora Schopf, Wilson \& Bentall 1944

Calamospora hartungiana Schopf in Schopf, Wilson \& Bentall

1944

Calamospora liquida Kosanke 1950

Gênero Punctatisporites Ibrahim emend. Potonié \& Kremp 1954

Punctatisporites gretensis Balme \& Hennelly 1956

Infraturma Apiculati Bennie \& Kidston emend. Potonié 1956

Subinfraturma Granulati Dybová \& Jachowicz 1957

Gênero Granulatisporites Ibrahim emend. Potonié \& Kremp

1954

Granulatisporites austroamericanus Archangelsky \& Gamerro 1979

Granulatisporites parvus (Ibrahim) Schopf, Wilson \& Bentall

1944

Gênero Cyclogranisporites Potonié \& Kremp 1954

Cyclogranisporites microgranulatus Menéndez \& Azcuy

emend. Archangelsky \& Gamerro 1979

Cyclogranisporites minutus Bharadwaj 1957

Subinfraturma VerRUCATI Dybová \& Jachowicz 1957

Gênero Verrucosisporites Ibrahim emend. Smith 1971

Verrucosisporites microtuberosus (Loose) Smith \& Butterworth 1967

Subinfraturma NoDATI Dybová \& Jachowicz 1957

Gênero Apiculatasporites Ibrahim 1933 emend. Smith y

Butterworth 1967

Apiculatasporites parviapiculatus Azcuy 1975

Apiculatasporites caperatus Menéndez \& Azcuy 1969

Gênero Apiculatisporis Ibrahim 1933 emend. Potonié \& Kremp 1956

Gênero Plicatipollenites Lele 1964

Plicatipollenites malabarensis (Potonié \& Sah) Foster 1975

Plicatipollenites densus Srivastava 1970

Gênero Potonieisporites Bharadwaj emend. Bharadwaj 1964

Potonieisporites novicus Bharadwaj emend. Poort \& Veld 1997

Potonieisporites barrelis Tiwari 1965

Potonieisporites magnus Lele \& Karim 1971

Gênero Caheniasaccites Bose \& Kar emend. Azcuy \&Di Pasquo

2000

Caheniasaccites flavatus (Bose \& Kar) emend. Azcuy \& Di

Pasquo 2000

Infraturma STRIASACCITI Bharadwaj 1962

Gênero Meristocorpus Playford \& Dino 2000

Meristocorpus explicatus Playford \& Dino 2000

Subturma Disaccites Cookson 1947
Apiculatisporis aculeatus (Ibrahim ) Potonié \& Kremp 1955

Subinfraturma BACULATI Dybová \& Jachowicz 1957

Gênero Horriditriletes Bharadwaj \& Salujha 1964

Horriditriletes uruguaiensis (Marques-Toigo) Archangelsky \&

Gamerro 1979

Subturma ZONOTRILETES Waltz 1935

Infraturma Cingulati Potonié \& Klaus emend. Dettmann 1963

Gênero Reticulatisporites Ibrahim emend. Neves 1964

Reticulatisporites pseudopalliatus Staplin 1960

Suprasubturma LAMINATITRILETES Smith \& Butterworth 1967

Subturma ZonolaminatitriLETES Smith \& Butterworth 1967

Infraturma CinguliCAVATI Smith \& Butterworth 1967

Gênero Lundbladispora Balme emend. Playford 1965

Lundbladispora braziliensis (Pant \& Srivastava) Marques-

Toigo \& Pons emend. Marques-Toigo \& Picarelli 1984

Lundbladispora riobonitensis Marques-Toigo \& Picarelli 1984

Gênero Vallatisporites Hacquebard 1957

Vallatisporites ciliaris (Lüber) Sullivan 1964

Vallatisporites arcuatus (Marques-Toigo) Archangelsky \& Gamerro 1979

Vallatisporites spinosus Cauduro 1970

Gênero Cristatisporites Potonié \& Kremp emend. Butterworth , Jansonius, Smith, Staplin 1964

Cristatisporites indignabundus (Loose) Potonié \& Kremp,

emend. Staplin \& Jansonius 1964

Cristatisporites crassilabratus (Archangelsky \& Gamerro

1979)

Suprasubturma Pseudosaccititriletes Richardson 1965

Infraturma MonopseudosACCITI Smith \& Butterworth 1967

Gênero Spelaeotriletes Neves \& Owens 1966

Spelaeotriletes ybertii (Marques-Toigo) Playford \& Powis

emend. Playford, Dino \& Marques-Toigo 2001

Anteturma VARIEGERMINANTES Potonié 1970

Turma SACCITES Erdtmann 1947

Subturma Monosaccites Chitaley emend. Potonié \& Kremp 1954

Infraturma DiPOLSACCITE Hart emend. Dibner 1971

Gênero Scheuringipollenites Tiwari 1973

Infraturma DisAcCitrileti Leschik emend. Potonié 1958

Scheuringipollenites maximus (Hart) Tewari 1973

Scheuringipollenites ovatus (Balme \& Hennelly) Foster 1975

Gênero Limitisporites Leschik emend. Schaarschmidt 1963

Limitisporites rectus Leschik 1956

Limitisporites luandensis Bose \& Maheshwari 1968

Divisão CHLORoPhyta Pascher 1914

Classe ChlorophyceaE Kützing 1843

Ordem Chlorococcalles Marchand emend. Pascher 1915

Família Botryococcaceae Wille 1909

Gênero Botryococcus Kützing 1849

Botryococcus braunii Kützing 1849 
Tabela 4 - Distribuição quantitativa das espécies que compõem as assembléias macro e mesoflorísticas.

\begin{tabular}{|c|c|c|}
\hline Millan 1972 a 1987 & Listagem atual & $\mathrm{n}^{\mathrm{o}}$ de espécimes \\
\hline Lepidodendron pedroanum & Leptophloeum cf. L. sanctae-helenae & 1 \\
\hline Lycopodiopsis pedroanus (pars) / B. pedroanum (pars) & Brasilodendron pedroanum & 7 \\
\hline$* * *$ & Aff. Brasilodendron sp & 1 \\
\hline Lycopodiopsis pedroanus (pars) / B. pedroanum (pars) & Bumbudendron cf. B. paganzianum & 3 \\
\hline $\begin{array}{l}\text { Lycopodiopsis sp Tipo A; Lycopodiopsis pedroanus } \\
\text { (pars) / B. pedroanum (pars) }\end{array}$ & Bumbudendron millanii & 3 \\
\hline Lycopodiopsis derby & Cf. Cyclodendron sp & 3 \\
\hline Sphenophyllum sp Tipo A (pars) & Trizygia $\mathrm{sp}$ & 1 \\
\hline Sphenophyllum sp Tipo A (pars) & Sphenophyllales indeterminadas & 4 \\
\hline Paracalamites cf. levis (pars) & Koretrophyllites sp & 4 \\
\hline Paracalamites australis (pars) & Paracalamites australis & 8 \\
\hline Paracalamites cf. levis (pars) & Paracalamites levis & 10 \\
\hline Paracalamites montemorensis (pars) & Paracalamites montemorensis & 12 \\
\hline Paracalamites australis (pars) & Paracalamites sp & 1 \\
\hline Nothorhacopteris cf. ovata & Cf. Nothorhacopteris sp A & 3 \\
\hline Nothorhacopteris cf. chubutiana & Cf. Nothorhacopteris sp B & 2 \\
\hline Botrychiopsis cf. plantiana; Adiantites sp & Botrychiopsis cf. B. plantiana; & 4 \\
\hline ? Botrychiopsis cf. weissiana & Cf. Botrychiopsis sp & 2 \\
\hline Cf. Ginkgophyllum sp A & Ginkgophyllum cf. G. diazii & 10 \\
\hline$* * *$ & Ginkgophyllum cf. G.kidstonii & 1 \\
\hline$* * *$ & (?) Ginkgophyllum spatulifolia & 1 \\
\hline Noeggerathiopsis hislopii & Noeggerathiopsis cf. N. hislopii & 6 \\
\hline Paranocladus? fallax & Paranocladus dusenii & 14 \\
\hline Buriadia heterophylla & Buriadia aff. B. heterophylla & 5 \\
\hline Cordaicarpus barbosanus & Cordaicarpus barbosanus & 1 \\
\hline$* * *$ & Cordaicarpus cesarii & 1 \\
\hline$* * *$ & Cordaicarpus sp & 3 \\
\hline Cordaicarpus nitens (pars) & Paranospermum cambuiense & 34 \\
\hline Cordaicarpus nitens (pars) & Paranospermum millanianum & 8 \\
\hline$* * *$ & Samaropsis barcellosa & 1 \\
\hline$* * *$ & Samaropsis cf. S. cuerdai & 1 \\
\hline Samaropsis seixasi & Samaropsis aff. S. seixasii & 2 \\
\hline
\end{tabular}

porites scutiformis Trindade 1970, Sublagenicula tripartites (Trindade 1970) nov. comb. e Setosisporites sp são típicos do Pensilvaniano brasileiro, enquanto as demais adentram o Eopermiano gondvânico (Tab. 5). As formas megaspóricas esfenofíticas estão representadas por um só gênero: Calamospora sp. figura $10(\mathrm{O})$. No documentário macroflorístico, todavia, as esfenófitas aparecem um pouco mais diversificadas, com formas de esfenofilales e equisetales, figura 9 (L e N).
A análise palinológica revelou uma composição predominantemente esporofítica, apresentando maior abundância de esporos relacionados às filicófitas e / ou progimnospermopsidas, e menor relativa às licófitas e esfenófitas, figura $11(\mathrm{~A}-\mathrm{N})$ e figura 12.

Quanto à tafonomia A presença de tétrades de esporos reforça a idéia de pouco transporte, figura 11 $(\mathrm{L}-\mathrm{M})$. Os grãos de pólen relacionados às coníferas 

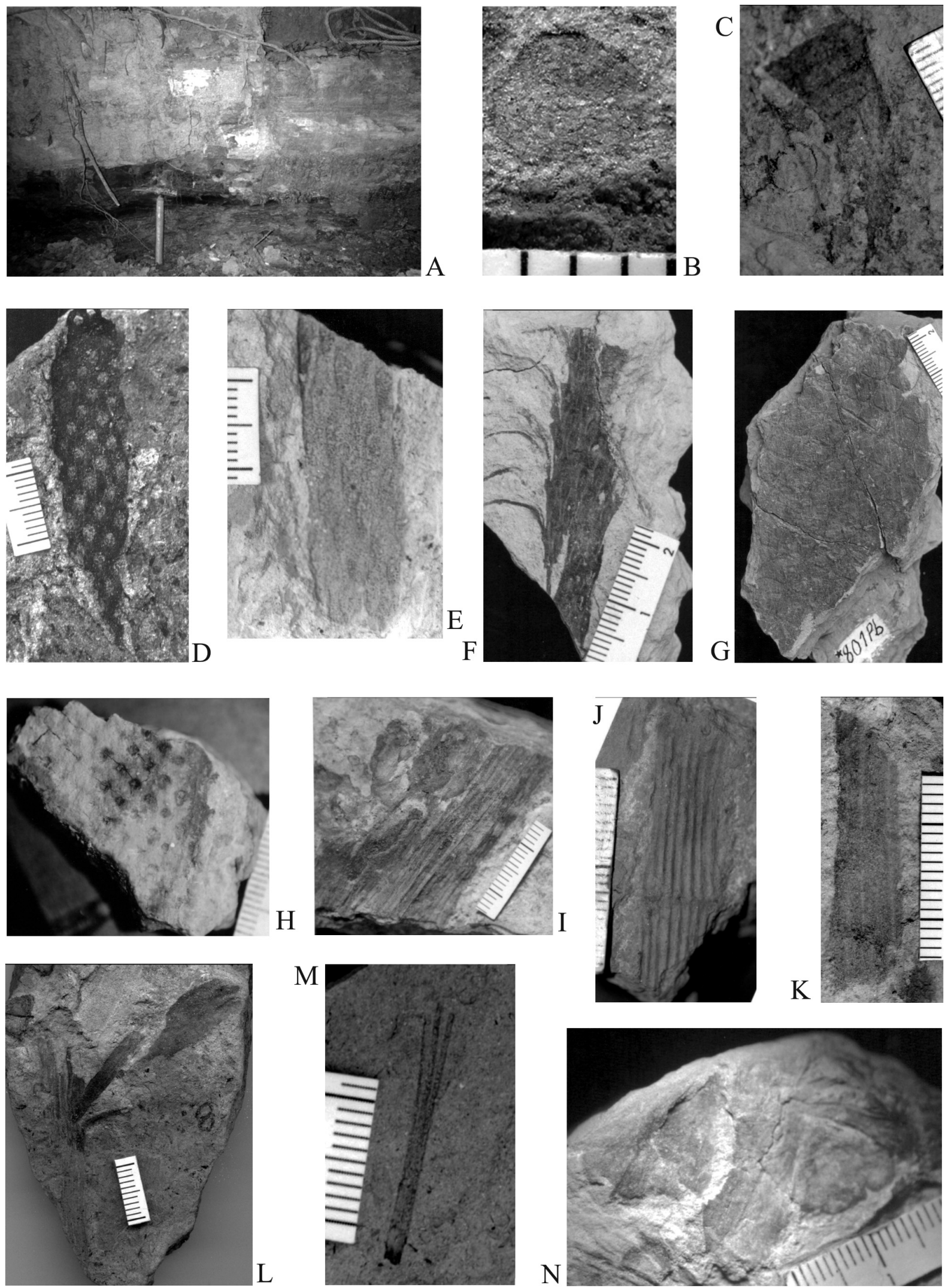

Figura 9 - Detalhe do afloramento e alguns macrofósseis e macrofitofósseis encontrados na Tafoflora de Monte Mor (SP). (A) Fina camada de carvão na base do afloramento; (B) Conchostráceo Leaia sp; (C) Euripterídeo encontrado na assembléia estudada por Millan (1972); (D) Brasilodendron pedroanum, GP3E 9135; (E) Bumbudendron millanii, GP/3E 9137; (F) Bumbudendron cf. B. paganzianum, $D G P$ MN 1088 Pb; (G) Leptophloeum cf. L. sanctae-helenae,DGP MN 801 Pb; (H) cf. Cyclodendron sp,DGP MN 1093 Pb; (I) Paracalamites levis, GP/3E 9264; (J) Paracalamites australis DGP MN $703 \mathrm{~Pb}$; (K) Paracalamites montemorensis, GP/3E 9266; (L) Koretrophyllites sp, GP/3E 9223; (M) Ginkgophyllum cf. G. diazii, GP/3E 9073a; (N) Trizygia sp, DGP MN 1079 Pb. 
e cordaitales, figura 11 (F-I) apresentam-se diversificados dentro da assembléia, não tendo porém maior significado paleoambiental, visto tratarem-se de grupos vegetais de grande capacidade de dispersão e produ- ção. Por outro lado, evidenciam que as coníferas recém surgidas no cenário mundial, já estariam relativamente bem estabelecidas nessa área.

Os macrofósseis de ramos caulinares de Para-

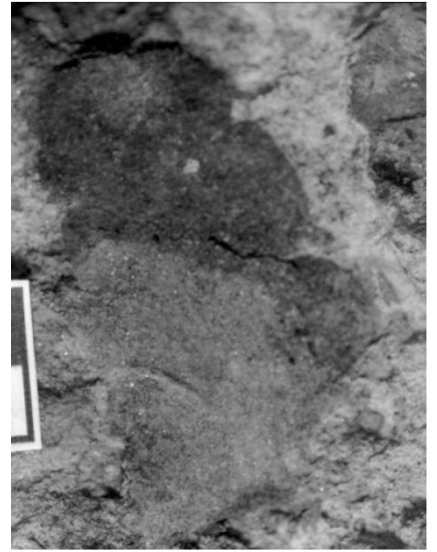

A
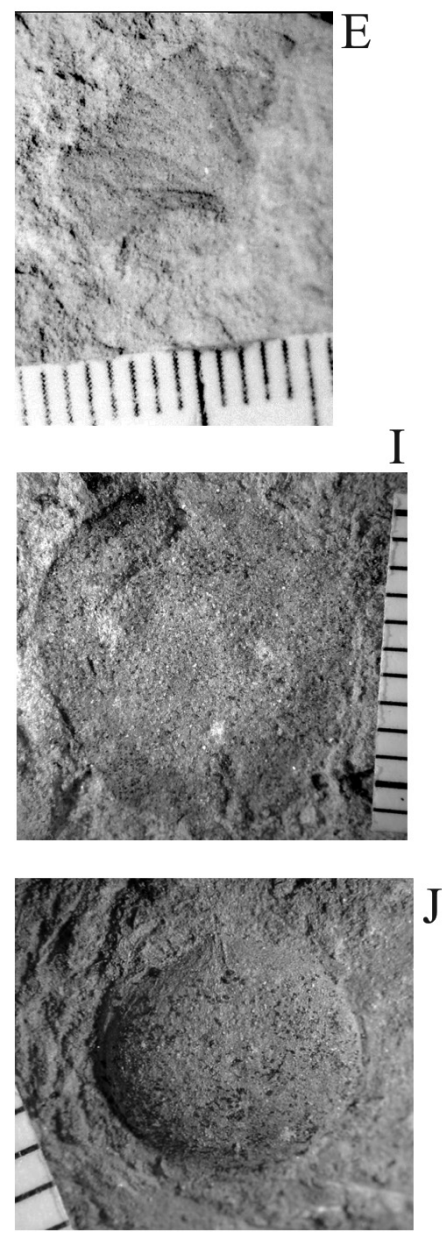
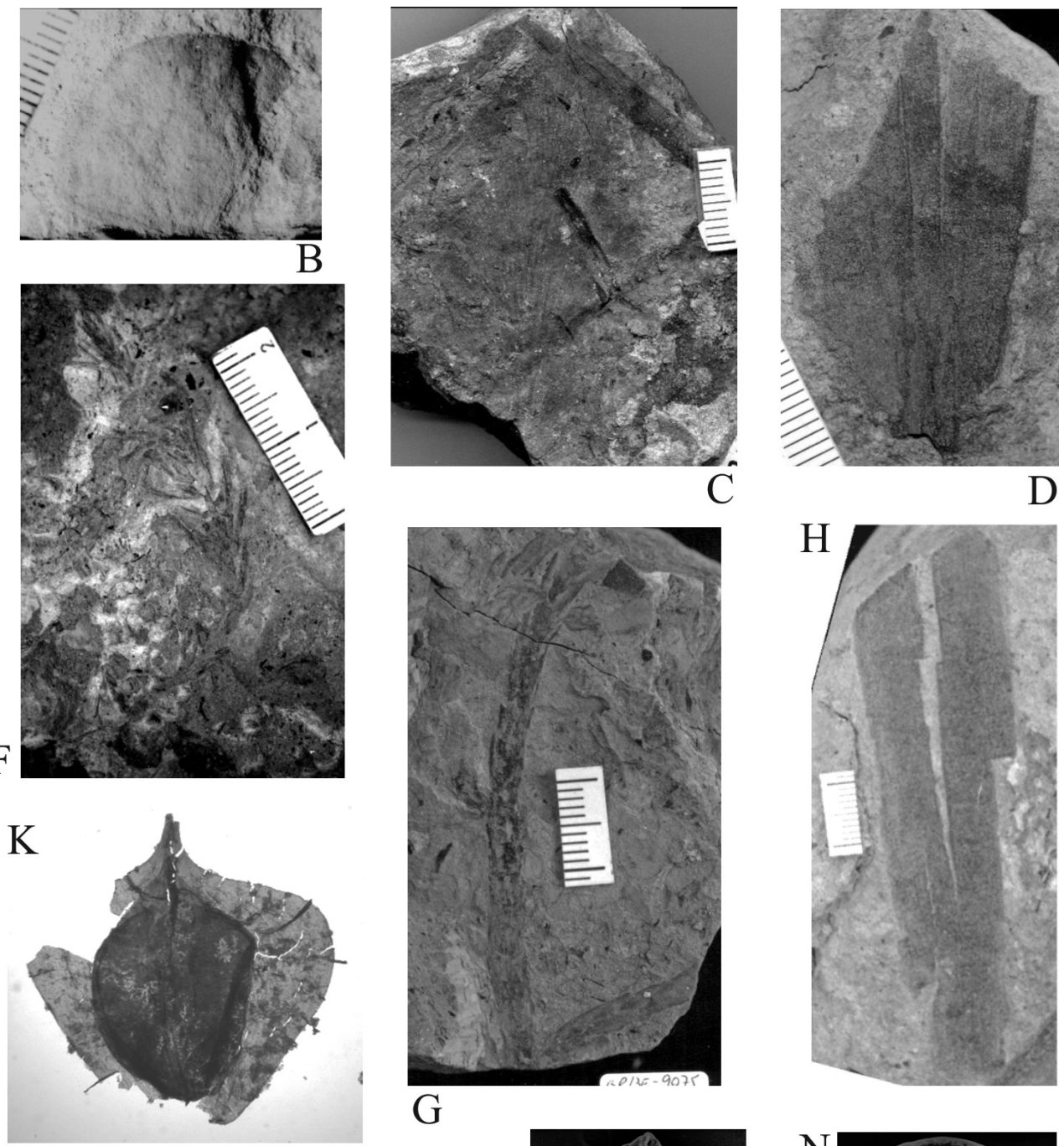

G

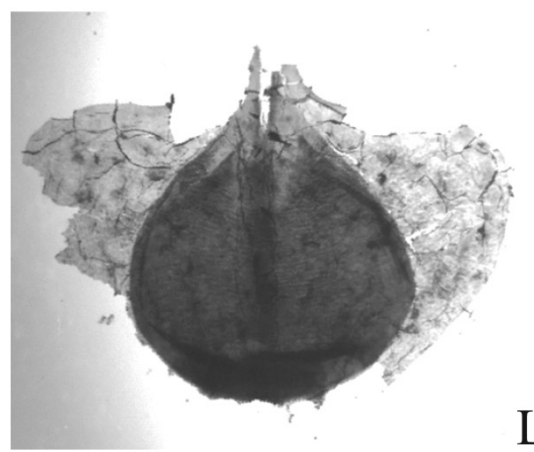

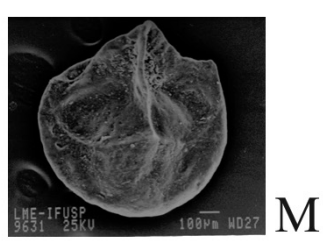
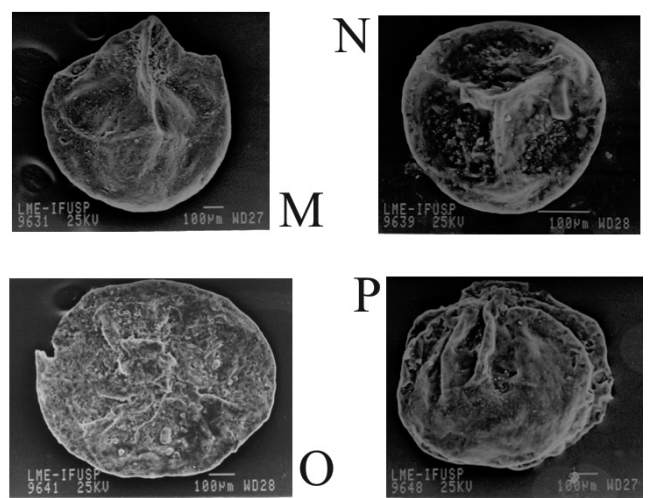

Figura 10 - Elementos macroflorísticos e mesoflorísticos da Tafoflora de Monte Mor (SP). (A) Botrychiopsis cf. B. plantiana, GP/3E 9107 aB; (B) cf. Nothorhacopteris sp A, DGP MN 1082 Pb; (C) (?) Ginkgophyllum spatulifolia, GP/3E 9107 b; (D) Ginkgophyllum cf. G. kidstonii, GP/3E 9099 a; (E) cf. Nothorhacopteris sp B, DGP MN 1084 Pb; (F) Buriadia aff. B. heterophylla, GP/3E 9147 a; (G) Paranocladus dusenii, GP/3E 9075; (H) Noeggerathiopsis cf. N. hislopii, GP/3E 9100 a; (I) Samaropsis cf. S. cuerdai, GP/3E 9224; (J) Cordaicarpus cesarii, GP/3E 9231 B; (K) Paranospermum cambuiense, lam. GP/3E 9192; (L) Paranospermum millanianum sp nov., lam. GP/3E 9197; (M) Sublagenicula brasiliensis, lam. GP/3E 9172, stub A (90 ${ }^{\circ}$ SE); (N) Banksisporites vulga-

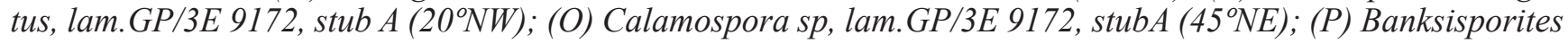
tenuis, lam. GP/3E 9172, stub B $\left(60^{\circ} \mathrm{SW}\right)$. 
calamites, figura 9 (I-K), coníferas e sementes aparecem, mais freqüentemente, associados na camada síltico-arenosa logo acima da capa do carvão, com preservação baixa ou nula de partes menos resistentes (folha). Esta associação pode ter sofrido certo transporte, com direção preferencial de $20^{\circ} \mathrm{NE}$ a $40^{\circ} \mathrm{NE}$, e depositadose em ambiente de planície de inundação. No caso das licófitas da lapa do carvão, o transporte pode ter sido baixo ou praticamente nulo. Isso é evidenciado por uma associação quase pura de licófitas com a preservação de seus caules de forma aleatória ou pouco orientados.

Aspectos paleoecológicos $\mathrm{Na}$ assembléia fitofossilífera proveniente da lapa e da capa do carvão, encontram-se elementos relacionados aos ambientes: hidrohigrófilo, higro-mesófilo e meso-xerófilo.

As licófitas da lapa do carvão estariam constituindo comunidades hidro-higrófilas arbustivas em associações puras, provavelmente, relacionadas a planícies de maré ou a corpos de água salobra.

Por outro lado, as licófitas da capa do carvão aparecem em associações mistas com esfenófitas, coníferas e sementes, denotando comunidades hidro-higrófilas a higro-mesófilas de planície de inundação ou em margens de canais interdistributários de planícies deltaicas. Nesse cenário, as terras baixas de planície deltaica e de planície de inundação seriam ocupadas por esfenófitas tipo Trizygia, em seus estratos hidrohigrófilos. Entretanto, as Koretrophyllites talvez ocupassem ambientes menos úmido das planícies fluviais (higro-mesófilas) junto a Botrychiopsis e Nothorhacopteris em associações tipo florestas interglaciais frias. No contexto mesófilo, apareceriam as Cordaitales e no meso-xerofílico, mais distante, as (?) Ginkgoales e as coníferas tipo Voltziales.

Aspectos paleoflorísticos e bioestratigráficos A tafoflora pensilvaniana do sítio Volpe desenvolveu-se em um contexto paleoflorístico de província gondvânica ocidental.

No contexto bioestratigráfico, foi definida anteriormente, com base em sua composição macro e mesoflorística, como Assembléia Fitofossilífera -Tipo para a Tafoflora "A" de Rösler (1978) e para o Piso Montemorense de Millan (1987).

Após esse estudo, integra e caracteriza a III associação megaflorística Paranocladus-GinkgophyllumBrasilodendron de Bernardes-de-Oliveira et al. (2005) (Fig. 13). Por sua composição microflorística, segundo Souza \& Marques -Toigo (2003), corresponde à Zona Intervalo Ahrensisporites cristatus. Entretanto, com base em novas análises onde foi constatada a presença, entre outros elementos, de Scheuringipollenites maximus (Hart) Tewari 1973, figura 11 (J - K), uma possível atribuição à Palinozona Intervalo Crucisaccites monoletus não pode ser descartada.

Em relação à fitoestratigrafia argentina, alguns elementos, tais como Bumbudendron millanii (Arrondo \& Petriella) Arrondo \& Petriella 1985, Bumbudendron cf. B. paganzianum, sugerem correlação com a Zona NBG. Entretanto, poderiam tratar-se de formas relictuais na paleoflora de Monte Mor, uma vez que a presença bem definida de coníferas Voltziales (Paranocladus e Buriadia), a possível presença de Botrychiopsis plantiana Archangelsky \& Arrondo 1971, a presença de sementes tipo Samaropsis cf. S. cuerdai (com estratotipo na Fm. Andapaico da Argentina) e Paracalamites levis Rigby 1966 emend Zampirolli \& Bernardes-deOliveira 2000 , sugerem melhor posicionamento bioestratigráfico na correlação com a Zona Intervalo de Archangelsky \& Cúneo (1991). Outros elementos como Ginkgophyllum cf. G. diazii, Paracalamites australis, Cordaicarpus cesarii se distribuem entre ambas as zonas (Fig. 13 e Tab. 6).

\section{Aspectos paleogeográficos e paleoclimáticos A flora}

Tabela 5 - Distribuição geográfica e cronológica das formas megaspóricas de Monte Mor encontradas por Trindade (1970), complementadas seguindo nomenclatura de Glasspool (2003).

\begin{tabular}{l|l|l}
\hline \multicolumn{1}{c|}{ Espécies } & \multicolumn{1}{c}{$\begin{array}{c}\text { Pensilvaniano } \\
\text { (Bashkiriano-Gzeliano) }\end{array}$} & $\begin{array}{c}\text { Cesuraliano } \\
\text { (Asseliano-Kunguriano) }\end{array}$ \\
\hline Calamospora sp & América do Sul (Br) & América do Sul, África Central ? \\
\hline Banksisporites endosporitiferus & América do Sul & América do Sul, África do Sul, África Central e Índia \\
\hline Banksisporites tenuis & América do Sul (Bre Arg.) & América do Sul, África Central ? \\
\hline Banksisporites vulgatus & América do Sul & América do Sul e Índia \\
\hline Banksisporites indicus & América do Sul & América do Sul e Índia \\
\hline Banksisporites labiosus & América do Sul & América do Sul \\
\hline Lagenoisporites scutiformis & América do Sul (Br) & \\
\hline Sublagenicula brasiliensis & América do Sul (Br e Arg.) & América do Sul, África do Sul e África Central ? \\
\hline Sublagenicula tripartites & América do Sul (Br) & \\
\hline Setosisporites sp & América do Sul (Br) & \\
\hline
\end{tabular}




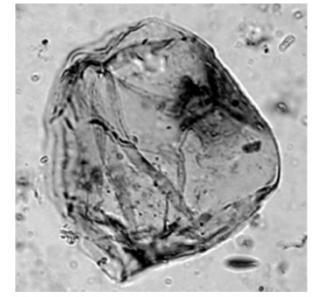

A

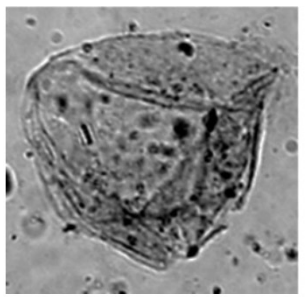

$\mathrm{B}$
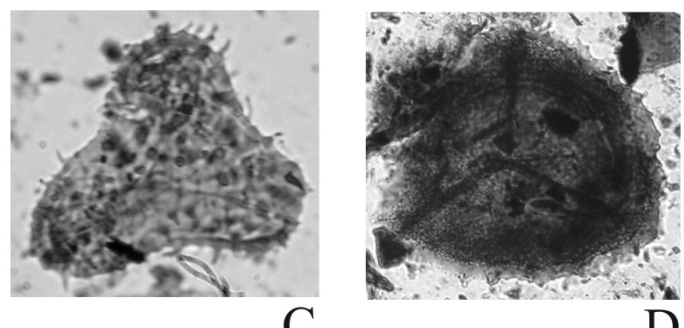

$\mathrm{D}$

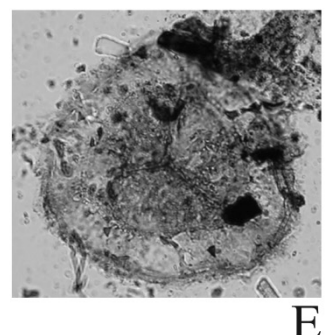

$\mathrm{E}$

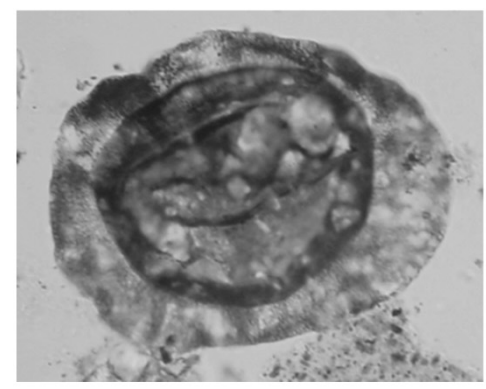

$\mathrm{F}$
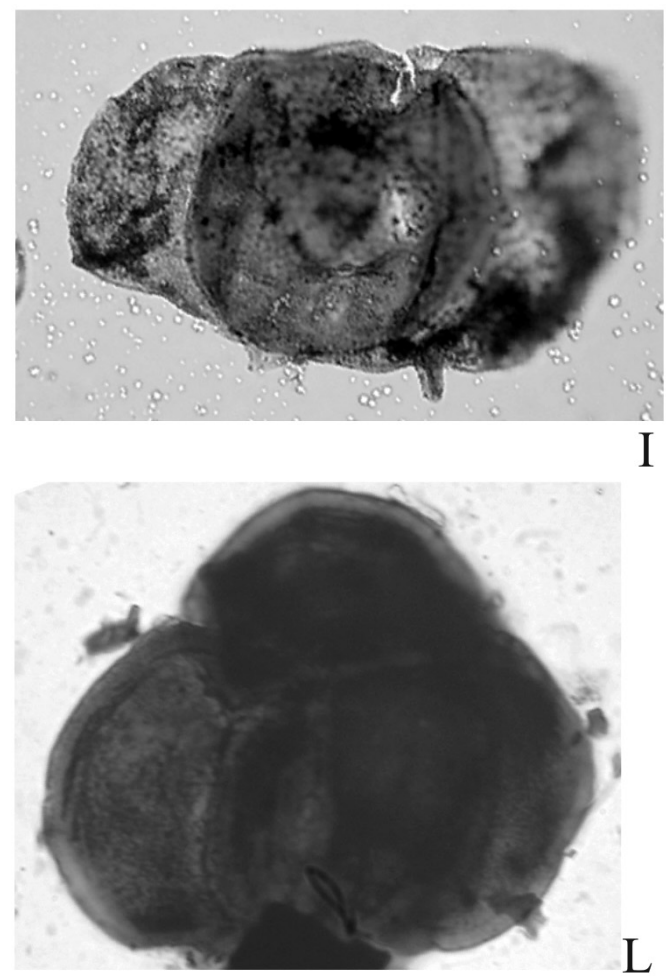

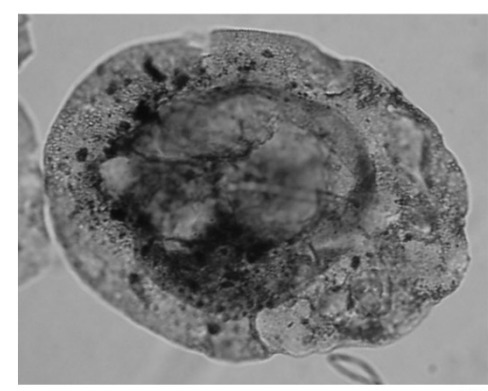

$\mathrm{G}$
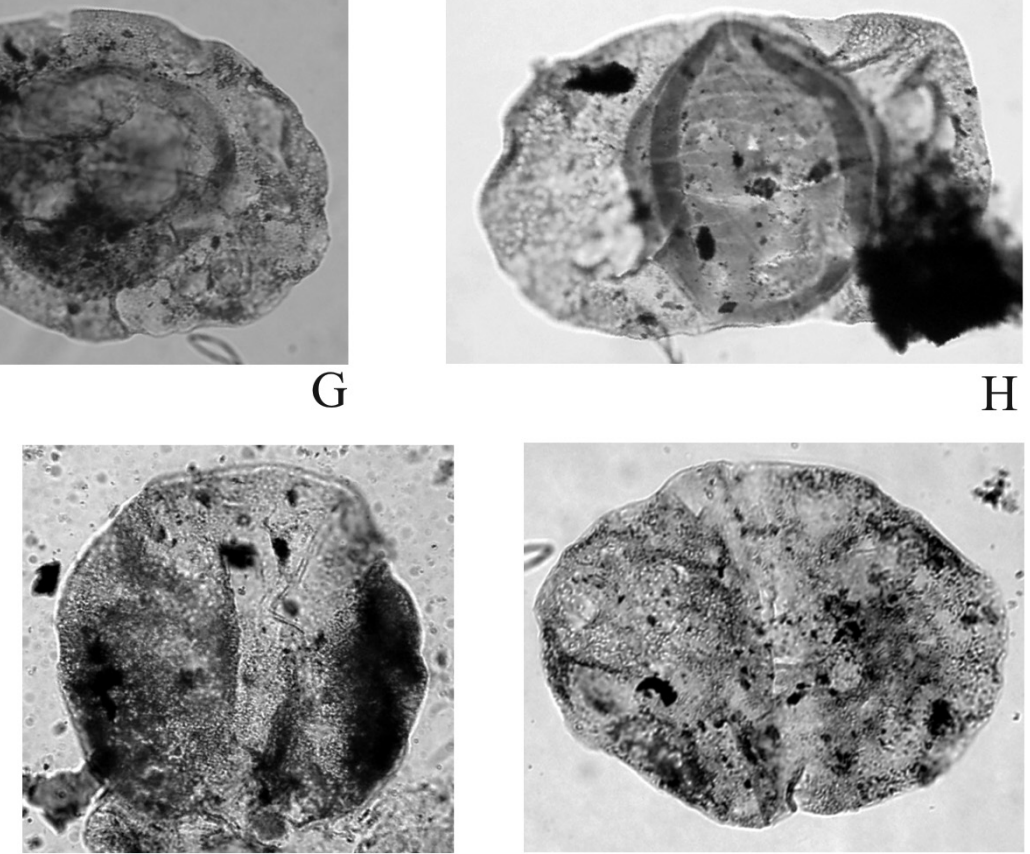

$\mathrm{H}$

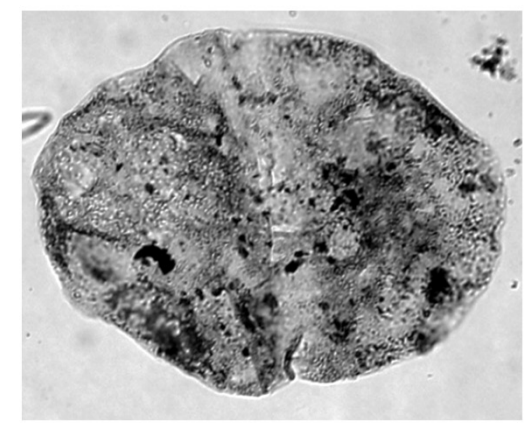

\section{(1)}
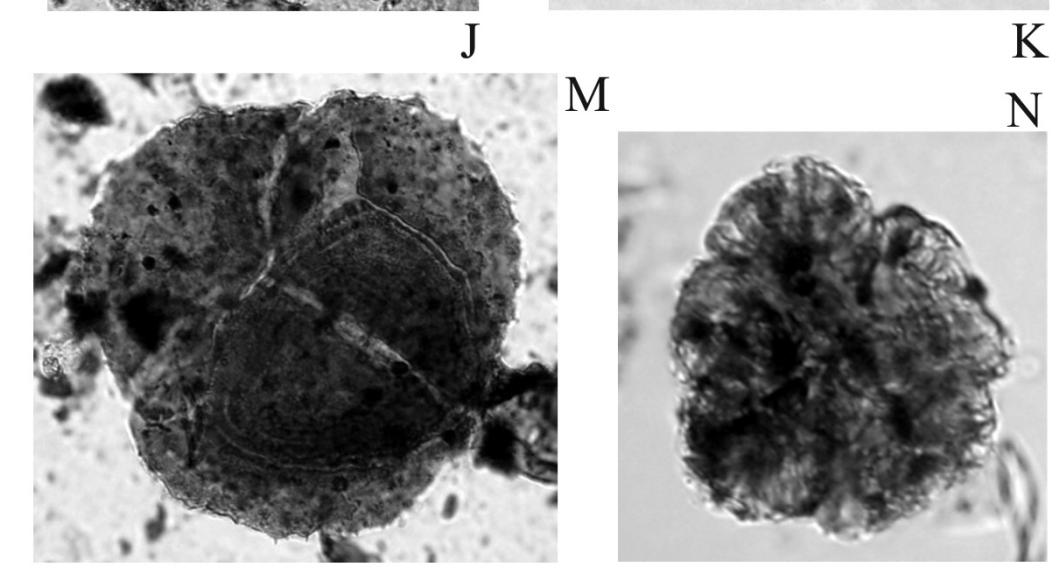

Figura 11 - Elementos microflorísticos da Tafoflora de Monte Mor (SP). (A) Calamospora hartungiana, lam. IG-P 383 B, K49/3-4 (63x imersão); (B) Calamospora liquida, lam. IG-P 384 A, T42/1 (63x imersão); (C) Horriditriletes uruguaiensis, IG-P 383 A, X 32/3 (63x imersão); (D) Lundbladispora braziliensis, lam. IG-P 383 A, Q 31/4 (63x imersão); (E) Vallatisporites ciliaris, lam. IG-P 383 A, Q 52 1/2 (63x imersão); (F) Plicatipollenites malabarensis, lam. IG-P 385 A, M43/3 (40x); (G) Potonieisporites novicus, lam. GP/3E, Res. E, lam.1,R 46/1 (63x imersão); (H) Meristocorpus explicatus, lam. GP/3E 9203, Res. E, lam.2, O48/3 (63x imersão); (I) Limistisporites rectus, GP/3E 9204, Res. F, lam.1, S46/3 (63x imersão); (J) Scheuringipollenites maximus, lam. IG-P 384 A, N43 (63x imersão); (K) Scheuringipollenites maximus, GP/3E 9203, Res. E lam.1, F35/1 (63x imersão); (L) Tétrade de esporos, lam. IG-P 383B, S51/3-4 (40x); (M) Tétrade de esporos, lam. IG-P 384 B, S41/2 (63x imersão); (N) Botryococcus braunii, lam. IG-P 385 F, P43/2-4 (40x).

interglacial do sítio Volpe se desenvolveu numa latitude entre $30^{\circ}$ e $60^{\circ} \mathrm{S}$ (Scotese \& Mckerrow, 1990), em condições paleoclimáticas frias. Todavia, com a retirada da geleira e a provável proximidade do ambiente marinho, um clima mais ameno deve ter-se estabelecido, a ponto de desenvolver-se uma vegetação razoavelmente abundante, capaz de formar camadas de carvão ainda que não muito espessas. 
A presença de algas do tipo Botryococcus ca- uma interpretação de ambiente continental, figuras 9(B) racterísticas de água doce (Emberger, 1968 p.73; Font e 11(N).

Quer, 1985 p.147) e de conchostráceos, corroboraria

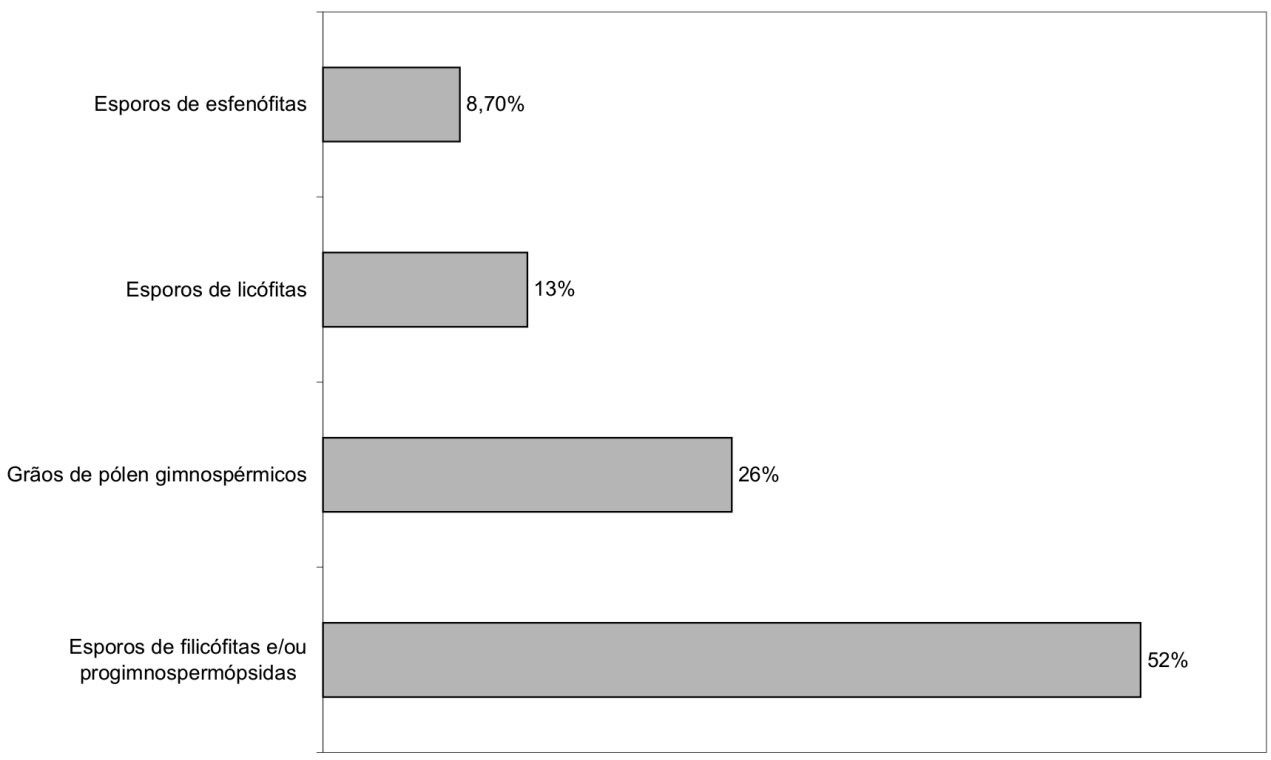

Figura 12 - Conteúdo palinológico da tafoflora do sítio Volpe, encontrado nas amostras analisadas por Mune (2005, inédito).

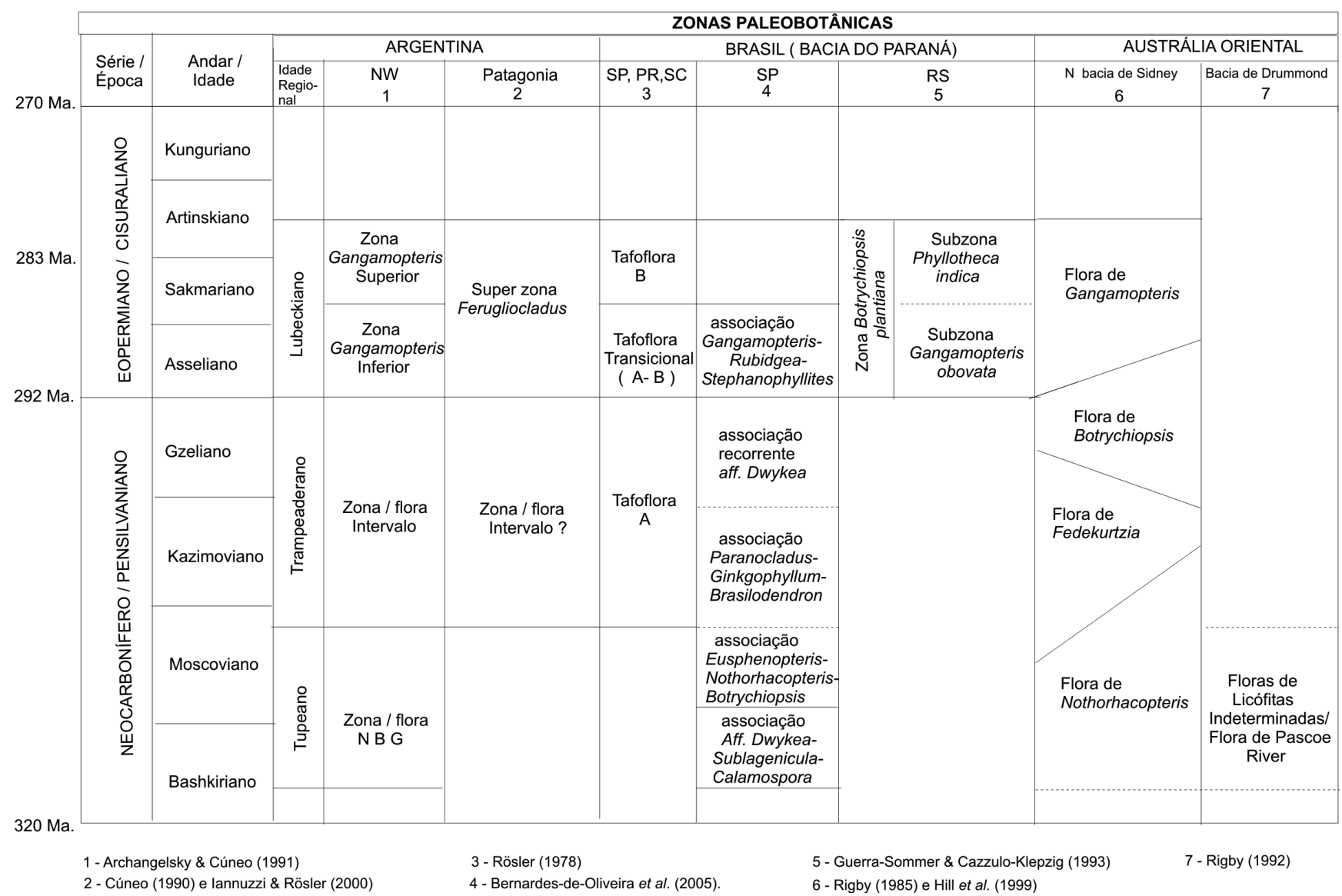

Figura 13 - Prováveis correlações entre associações megaflorísticas pensilvanianas gondvânicas, modificado de Bernardes-de-Oliveira et al. (2005). 
Tabela 6 - Espécies detectadas em Monte Mor que aparecem nas Zonas Florísticas NBG e Intervalo,segundo Archangelsky \& Cúneo (1991).

\begin{tabular}{|c|c|c|}
\hline Taxa presentes em Monte Mor & Zona NBG & Zona Intervalo \\
\hline BUMBUDENDRON MILLANII & $\mathrm{X}$ & \\
\hline Bumbudendron cf. B. paganzianum & $\mathrm{X}$ & \\
\hline \multicolumn{3}{|l|}{ LEPTOPHLOEUM CF. L. SANCTAE-HELENAE } \\
\hline BRASILODENDRON PEDROANUM & $\mathrm{X}$ & \\
\hline Aff. Brasilodendron $\mathrm{sp}$ & $\mathrm{X}$ & \\
\hline \multicolumn{3}{|l|}{ Cf. Cyclodendron $\mathrm{sp}$} \\
\hline \multicolumn{3}{|l|}{ Trizygia $\mathrm{sp}$} \\
\hline \multicolumn{3}{|l|}{ Sphenophyllales indet. } \\
\hline \multicolumn{3}{|l|}{ Koretrophyllites sp } \\
\hline Paracalamites australis & $\mathrm{X}$ & $\mathrm{X}$ \\
\hline PARACALAMITES LEVIS & & $\mathrm{X}$ \\
\hline \multicolumn{3}{|l|}{ Paracalamites montemorensis } \\
\hline \multicolumn{3}{|l|}{ Paracalamites sp } \\
\hline Botrychiopsis ef. B. plantiana & & $\mathrm{X}$ \\
\hline \multicolumn{3}{|l|}{ Cf. Botrychiopsis sp B } \\
\hline \multicolumn{3}{|l|}{ Cf. Nothorhacopteris sp A } \\
\hline \multicolumn{3}{|l|}{ Cf. Nothorhacopteris sp B } \\
\hline \multicolumn{3}{|l|}{ NOEGGERATHIOPSIS CF. N. HISLOPII } \\
\hline GINKGOPHYLLUM CF. G. DIAZII & $\mathrm{X}$ & $\mathrm{X}$ \\
\hline \multicolumn{3}{|l|}{ Ginkgophyllum cf. G. kidstonii } \\
\hline \multicolumn{3}{|l|}{ (?) Ginkgophyllum spatulifolia } \\
\hline \multicolumn{3}{|l|}{ BURIADIA AFF. B. HETEROPHYLLA } \\
\hline \multicolumn{3}{|l|}{ PARANOCLADUS DUSENII } \\
\hline \multicolumn{3}{|l|}{ Samaropsis aff. S. seixasi } \\
\hline SAMAROPSIS CF. S. CUERDAI & & $\mathrm{X}$ \\
\hline \multicolumn{3}{|l|}{ Samaropsis sp } \\
\hline \multicolumn{3}{|l|}{ Samaropsis barcellosa } \\
\hline CORDAICARPUS CESARII & $\mathrm{X}$ & $\mathrm{X}$ \\
\hline \multicolumn{3}{|l|}{ Cordaicarpus sp } \\
\hline \multicolumn{3}{|l|}{ PARANOSPERMUM CAMBUIENSE } \\
\hline Paranospermum millanianum sp nov. & & \\
\hline
\end{tabular}

CONCLUSÕES Após a revisão detalhada da Tafoflora de Monte Mor, algumas conclusões se tornam evidentes: (1) Caracteriza-se pela predominância de plantas esporofiticas (esfenófitas e licófitas) e em menor quantidade por macrofósseis gimnospérmicos dentre os quais o primeiro registro de coníferas na bacia do Paraná; (2) Esta associação pode ter sofrido certo transporte, com direção preferencial de $20^{\circ} \mathrm{NE}$ a $40^{\circ} \mathrm{NE}$, e depositado-se em ambiente de planície de inundação;
(3) Na assembléia fitofossilífera proveniente da lapa e da capa do carvão, encontram-se elementos relacionados aos ambientes: hidro-higrófilo, higro-mesófilo e meso-xerófilo; (4) A tafoflora pensilvaniana do sítio Volpe desenvolveu-se em um contexto paleoflorístico de província gondvânica ocidental, integrando e caracterizando a III associação megaflorística Paranocladus-Ginkgophyllum-Brasilodendron de Bernardes-deOliveira et al. (2005); (5) Provavelmente, dada a pre- 
sença de Scheuringipollenites maximus (Hart) Tewari 1973, pertencente a Palinozona Intervalo Crucisaccites monoletus; a presença bem definida de coníferas Voltziales (Paranocladus e Buriadia); a possível presença de Botrychiopsis plantiana Archangelsky \& Arrondo 1971; a presençade sementes tipo Samaropsis cf. $S$. cuerdai (com estrato-tipo na Fm. Andapaico da Argentina) e Paracalamites levis Rigby 1966 emend Zampirolli \& Bernardes-de-Oliveira 2000 , sugerem melhor posicionamento bioestratigráfico na correlação com a Zona Intervalo de Archangelsky \& Cúneo (1991); (6) um clima mais ameno deve ter-se estabelecido, a ponto de desenvolver-se uma vegetação razoavelmente abundante, capaz de formar camadas de carvão ainda que não muito espessas.
Agradecimentos Ao Programa de Pós-Graduação em Geologia Sedimentar do IGc/USP; à FAPESP, pelo apoio financeiro através do Projeto Temático "Levantamento da composição e sucessão paleoflorísticas do Neocarbonífero-Eopermiano (Grupo Tubarão), no Estado de São Paulo"; ao Museu Nacional, através da Dra. Diana Mussa (in Memorian) e Dr. Marcelo de Araújo Carvalho pelo acesso concedido às visitas técnicas à coleção paleobotânica de Monte Mor; ao Laboratório de Microscopia Eletrônica do Instituto de Física - IF/USP, para a obtenção de fotos em M.E.V e ao Dr. Fernando C. Fittipaldi pelas aulas de preparação de cutículas do Paleozóico.

\section{Referências}

Alarcon C.M. \& Bernardes-de-Oliveira M.E.C. 1999. Licófitas Neopaleozóicas da Bacia do Paraná (Brasil). I - Avaliação estratigráfica geral, com ênfase em Lycopodiopsis derbyi Renault. Revista Universidade Guarulhos, Série Pós Graduação, 1:35-49.

Archangelsky S. \& Cúneo R. 1991. The Neopaleozoic Floristic Succession from Northwestern Argentina. A New Perspective. In: Internacional Gondwana Symposium, 7 , São Paulo, atas, p. 469-481.

Arrondo O.G. \& Petriella B. 1985. Bumbudendron millani (Arrondo et Petriella) n. comb., del Carbonico-Permico de Argentina y Brasil. Rev. Ameghiniana, 21(2-4):169171.

Barbosa O. \& Almeida F.F.M. 1949. A Série Tubarão na Bacia do Rio Tietê, Estado de São Paulo. Notas Preliminares e Estudo. Rio de Janeiro, DNPM, Boletim 48 p.1-16.

Barbosa O. \& Gomes F.A. 1958. Pesquisa de petróleo na Bacia do rio Corumbataí, Estado de São Paulo. Rio de Janeiro, DNPM, Boletim 171, p.1-40.

Bernardes-de-Oliveira M.E., Rohn R., Ricardi-Branco F., Zampirolli A.P., Mune S.E., Amaral P.G.C., Longhim M.E., Castro Fernandes M.C., Lages L. 2005. Late Carboniferous to Early Permian glacial related paleofloras from northeastern Paraná Basin, Brazil. In: Gondwana Symposium, 12, Mendoza, Academia Nacional de Ciencias, atas, p.70.

Cuneo R. 1990. La tafoflora de la Formacion Mojon de Hierro (Grupo Tepuel) en la localidad Arroyo Garrido, Paleozoico Superior, Provincia de Chubut. Rev. Ameghiniana, 27(3-4):225-238.

Emberger L. 1968. Les plantes fossils- dans leurs rapports avec les végétaux vivants. Masson \& Cie, Editeurs, Paris. $758 \mathrm{p}$.

Fittipaldi F.C. \& Rösler O. 1978. Paranocladus ? fallax (conífera) estudos cuticulares. Bol. IG/USP., 9:109-113.

Font Quer P. 1985. Diccionario de Botánica. $9^{\mathrm{a}}$ ed., Barcelona, Editorial Labor, 1244 p.

França A. B. \& Potter P. R. 1988. Estratigrafia, ambiente deposicional e análise de reservatório do Grupo Itararé (Permocarbonífero), Bacia do Paraná (Parte 1). Boletim de Geociências da Petrobrás, 2(2/4):147-191.
Glasspool I.J. 2003. A review of Permian Gondwana megaspores, with particular emphasis on material collected from coals of the Witbank Basin of South Africa and the Sydney Basin of Australia. Review of Palaeobotany \& Palynology, 124 (3-4): 227-296.

Guerra-Sommer M. \& Cazzulo-Klepzig M.C. 1993. Biostratigraphy of the Southern Brazilian Neopaleozoic Gondwana sequence: a preliminary palaeobotanical approach. In: Congrès International de la Stratigraphie et Géologie du Carbonifère et Permien, 12, Buenos Aires, Comptes Rendus, v.2, p. 61-72.

Hill R.S., Truswell E.M., Mcloughlin S. \& Dettmann M.E. 1999. Evolution of the Australian Flora: Fossil Evidence. In: A.E. Orchard \& H.S. Thompson (eds.) Flora of Australia, Canberra. Australian Biological Resources Study, v. 1, p. 251-319.

Iannuzzi R. \& Rosler O. 2000. Floristic migration in South America during the Carboniferous: phytogeographic and biostratigraphic implications. Palaeogeographyc, $\mathrm{Pa}$ laeoclimatology, Palaeoecology, 161:71-94.

Milani J.E., França A.B., Schneider R.L. 1994. Bacia do Paraná. Boletim de Geociências da Petrobrás, 8(1):69-82.

Milani J.E., Facccini U.F, Scherer C.M., Araújo L.M., Cupertino J.A. 1998. Sequences and Stratigraphyc hierarchy of the Paraná Basin (Ordovician to cretaceous), Southern Brazil. Bol. IG/USP, 29:125-173.

Millan J.H. 1972. Macroflórula Carbonífera de Monte Mor, Estado de São Paulo. Tese de Doutoramento, Universidade de São Paulo, 165 p., 12 est.

Millan J.H. 1974. Sobre as Coniferopsidas da flórula gondvânica de Monte Mor, SP, Brasil. Rev. Ameghiniana, 11(2):124-134.

Millan J. H. 1975. Tafoflórula - Monte Mor, SP: Seus elementos e seu significado no gondwana inferior do Brasil. Rev. Bras. Geoc., 5(1):1-14.

Millan J.H. 1976. Moldes medulares da tafoflórula gondwânica de Monte Mor, Estado de São Paulo. Acad. Bras. Cien., Rio de Janeiro, Anais, 48(3):604.

Millan J.H. 1977a. Moldes Medulares da Tafoflórula Gondwânica de Monte Mor, Estado de São Paulo. Acad. Bras. Cien., Rio de Janeiro, Anais 49(1):195-204. 
Millan J.H. 1977 b. A macroflora do Gondwana brasileiro. Rio de Janeiro, Boletim Geográfico, 35(255):80-93.

Millan J.H. 1978. Rhacopteris e Botrychiopsis no Eogondwana de Monte Mor, Subgrupo Itararé, de SP. Acad. Bras. Cien. Rio de Janeiro, Anais, 50(3):427-428.

Millan J.H. 1979 a. Rhacopteris e Botrychiopsis no Eogondwana de Monte Mor, Subgrupo Itararé do Estado de São Paulo. Acad. Bras. Cien. Rio de Janeiro, Anais, 51(1):109-120.

Millan J.H. 1979 b. Catálogo prévio dos espécimes-tipos de sementes gondvânicas. Museu Nacional, Rio de Janeiro. Boletim Série Geologia, 37:1-10.

Millan J.H. 1980. Lepidodendrales do Eogondwana de Monte Mor, SP. Base do Subgrupo Itararé. I - Brasilodendron pedroanum (Carruthers) Chaloner, Leistikow \& Hill, 1979. Boletim do IG, USP, 11:105-113.

Millan J.H. 1981. Sobre a presença de folhas cf. Ginkgophyllum no Eogondwana de Monte Mor, Subgrupo Itararé do Estado de São Paulo. Museu Nacional, Rio de Janeiro, Boletim Série Geologia, 39:12.

Millan J.H. 1985. Lepdodrendales do Eogondwana de Monte Mor, SP, base do Subgrupo Itararé. 2- Sobre o gênero Lepidodendron Stern. In: Congresso Brasileiro de Paleontologia, 8, Rio de Janeiro, Resumo das comunicações, p. 106.

Millan J.H. 1987. Os pisos florísticos do carvão do Subgrupo Itararé no Estado de São Paulo e suas implicações. In: Congresso Brasileiro de Paleontologia, 10, Rio de Janeiro, Anais, p. 832-857.

Mune S.E. 2005. Tafoflora interglacial neocarbonífera do sítio Volpe, município de Monte Mor (SP), Subgrupo Itararé, nordeste da bacia do Paraná: Revisão e complementação. Dissertação de Mestrado, Instituto de Geociências, Universidade de São Paulo, 137 p., 13 est.

Petri S. \& Souza P.A. 1993. Síntese dos conhecimentos e nova concepção sobre a bioestratigrafia do Subgrupo Itararé, Bacia do Paraná, Brasil.Rev. IG. 14(1):7-18.

Quadros L.P. \& Melo J.H.G. 1987. Método prático de preparação palinológica em sedimentos do Pré-Mesozóico. Practical method of Palynologic Preparation of PréMesozoic Sediments. Bol. Geoc. PETROBRÁS, 1(2):205214

Read C.B. 1941. Plantas fósseis do Neo-Paleozóico do Paraná e Santa Catarina, Brasil. DNPM, Div. Geol. Min. Rio de Janeiro. Monografia 12, 102p.

Ricardi-Branco F.S.R.T. 1997. Tafoflora Gondvânica do Membro Triunfo, Formação Rio Bonito (Eopermiano), no Município de Figueira, Pr. Tese de Doutoramento,
Instituto de Geociências, Universidade de São Paulo, 182 p., 16 est.

Rigby J.F. 1966. The Lower Gondwana Floras of the Perth and Collie Basins, Western Australia. Palaeontographica, Abt. B, 118(4/6):113-152.

Rigby J.F. 1985. Aspects of Carboniferous paleobotany in eastern Australia. Dixième Congrès International de Stratigraphie et de Géologie du Carbonifère. Madrid. Compte Rendu, 4:307-312.

Rigby J.F. 1992. Plant remains from the Carboniferous Pascoe River beds of North Queensland. In: B.S. Venkatachala, K.P. Jain, N. Awasthi, (eds.) Proceedings of Birbal Sahni Birth Centenary Palaeobotanical Conference. Geophytology, 22:83-87.

Rösler O. 1978. The Brazilian eogondwanic floral succession. Boletim do Instituto de Geociências USP, 9:85-91.

Scotese C.R. \& Mckerrow W.S. 1990. Revised world maps and introduction. In: W.S. Mckerrow \& C.R. Scotese (eds.) Palaeozoic Palaeogeography and Biogeography. London, The Geological Society, Memoir 12, p.1-21.

Schneider R.L., Mühlmann H., Tomazi E., Medeiros R.A., Daemon R.F., Nogueira A.A. 1974. Revisão estratigráfica da Bacia do Paraná. In: Congresso Brasileiro de Geologia, 28, Porto Alegre, Anais, v.1, p. 41-86.

Souza Filho E.E. de. 1986. Mapeamento faciológico do Subgrupo Itararé na quadrícula de Campinas (SP). Dissertação de Mestrado, Instituto de Geociências, Universidade de São Paulo, 121 p.

Souza P.A. de. 2000. Palinobioestratigrafia do Subgrupo Itararé Carbonífero/Permiano, na porção Nordeste da Bacia do Paraná (SP/PR, Brasil). Tese de Doutorado, Instituto de Geociências, Universidade de São Paulo, $192 \mathrm{p}$.

Souza P.A. \& Marques-Toigo M. 2003. An overview on the Palynostratigraphy of the Upper Paleozoic strata of the brazilian Paraná Basin. Rev. Mus. Argentino Cienc. Nat,. 5(2):205-214.

Trindade N.M. 1970. Megásporos Carboníferos de Monte Mor, Estado de São Paulo. Acad. Bras. Ciências, Anais, 42(3):459-470.

Zampirolli A.P. \& Bernardes-de-Oliveira M.E.C. 2000. O gênero Paracalamites Zalessky, na tafoflora neocarbonífera de Itapeva (SP), Subgrupo Itararé, bacia do Paraná, Brasil. São Paulo. Revista do Instituto Geológico, 21(1/2):7-15.

Manuscrito A-1656 Aceito em 26 de junho de 2006 